\title{
An asymptotic analysis of the buckling of a highly shear-resistant vesicle
}

\author{
SYLVAIN REBOUX, GILES RICHARDSON and OLIVER E. JENSEN \\ School of Mathematical Sciences, University of Nottingham, University Park, \\ Nottingham NG7 2RD, UK \\ email: oliver.jensen@nottingham.ac.uk
}

(Received 5 June 2008; revised 16 May 2009)

\begin{abstract}
The static compression between two smooth plates of an axisymmetric capsule or vesicle is investigated by means of asymptotic analysis. The governing equations of the vesicle are derived from thin-shell theory and involve a bending stiffness $B$, a shear modulus $H$, the unstressed vesicle radius $a$ and a constant surface-area constraint. The sixth-order freeboundary problem obtained by a balance-of-forces approach is addressed in the limit when the dimensionless parameter $C=H a^{2} / B$ is large and the plate displacements are small. When the plate displacement is of order $a C^{-1 / 2}$, the vesicle undergoes a sub-critical buckling instability which is captured by leading-order asymptotics. Asymptotic linear and quadratic force-displacement relations for the pre- and post-buckled solutions are determined. The leading-order post-buckled solution is described by a simple fourth-order problem, exhibiting stress-focusing with stretching and bending confined to a narrow boundary layer. In contrast, in the pre-buckled state, stretching occurs over a larger length scale than bending. The results are in good qualitative agreement with numerical simulations for finite values of $C$.
\end{abstract}

\section{Introduction}

The mechanics of thin elastic shells under contact forces, a well-known topic in civil and mechanical engineering, has received considerable attention in recent years from the biotechnology community. Man-made micro- or nanocapsules have found a wide range of industrial applications (Kumar, 2000; Schrooyen et al., 2001; Monllor et al., 2007). Capsules and vesicles are also ubiquitous in living organisms, e.g. as biological cells, viruses or organelles and their mechanical properties mediate numerous biological processes. Understanding their behaviour under strain is crucial in analysing problems such as neutrophils or red blood cells squeezing through capillaries (Huang et al., 2001) or leukocyte rolling close to adhesive surfaces (Komura et al., 2005; Schwarz, 2007).

The static buckling and instability of a vesicle was described by Canham (1970) using an energy-minimising variational approach and by Zarda et al. (1977) and Pamplona \& Calladine (1993) using a balance-of-forces approach derived from thin-shell theory. Some discrepancies between the two approaches have been pointed out by Blyth \& Pozrikidis (2004) and it is still not clear how both views can be reconciled in a consistent manner. The most commonly used constitutive models for membranes with negligible bending stiffness are discussed by, e.g. Risso \& Carin (2004) and Wan et al. (2003); the difficulties 
in providing experimental validations for each of them are underlined in Smith et al. (1998).

Recently, Preston et al. (2008) formulated a sixth-order free-boundary problem to describe an axisymmetric permeable vesicle under compression between smooth parallel plates, using constitutive relations from Evans \& Skalak (1980), Pamplona \& Calladine (1993) and Parker \& Winlove (1999). As in most two-dimensional models derived from elastic thin-shell theory (Pamplona et al., 2005), the mechanical properties of the vesicle are found to depend on a single dimensionless parameter $C=H a^{2} / B$, where $H$ is the twodimensional in-plane shear modulus, $B$ the bending stiffness and $a$ the radius of the undeformed vesicle. Here, $C$ has been termed the Föppl-von Kármán (FvK) number in the context of viral capsids (Lidmar et al., 2003) and crumpling sheets (Lobkovsky \& Witten, 1997). In the context of a homogeneous linear elastic shell of radius $a$, thickness $h_{s}$ and Poisson ratio $v$ (with Love's first approximation; Landau \& Lifshitz, 1986; Pozrikidis, 2003), the FvK number can also be written as $C=12\left(1-v^{2}\right) a^{2} / h_{s}^{2}$. Note that $C$ does not depend on Young's modulus in this particular approximation. In applications where isotropic linear elasticity may not be appropriate, such as the lipid bilayer of a vesicle, it is more natural to characterise the shell by $H$ and $B$ directly.

Both Preston et al. (2008) and Pamplona et al. (2005) assume in-plane area incompressibility of the shell, a hypothesis particularly applicable to lipid bilayers. With this restriction, the stretching stiffness of the vesicle modelled as a two-dimensional surface is proportional to its resistance to shear (Evans \& Skalak, 1980). Vesicles or capsules with large values of $C$ are therefore highly stretch-resistant, in which case most bending and stretching can be expected to occur only in narrow regions, a phenomenon termed stress-focusing (Witten, 2007). Stretching and bending are typically confined to boundary layers of length scale $\left(a h_{s}\right)^{1 / 2}$ (Landau \& Lifshitz, 1986; Helfer et al., 2001), or, equivalently, $a C^{-1 / 4}$ (Lidmar et al., 2003). Pogoroelov (1986) pioneered a geometric theory for the buckling of shells assuming that deformations were isometric outside such thin singular regions.

Since the FvK number tends to infinity for very thin shells, it is not uncommon to encounter large values of $C$ : for example, $100<C<2000$ for spherical viruses prone to buckling (Lidmar et al., 2003); $C \approx 10^{3}$ for $15 \mu \mathrm{m}$ diameter vesicles made of self-assembled actin-coated membranes (Helfer et al., 2001) and $C \approx 100$ for red blood cells (Noguchi \& Gompper, 2005). This motivates the study of the asymptotic limit $C \rightarrow \infty$. In the context of buckling spherical shells under uniform or distributed loading, stretching and bending boundary layers were identified by Kriegsmann \& Lange (1980), Wan (1980), Parker \& Wan (1984), Graff et al. (1985), Scheidl \& Troger (1987) and Evkin \& Kalamkarov (2001) using Reissner's formulation, which involves a fourth-order description of an elastic shell. However, none of these studies used the constitutive assumptions adopted by Preston et al. (2008), nor did they account for the contact forces at the internal free boundary due to compression by a plate.

In the present paper, we examine the asymptotic behaviour for large $C$ of the freeboundary problem derived by Preston et al. (2008) for the compression of a vesicle between two plates as the inter-plate distance $h$ varies. Depending on the magnitude of the displacement relative to $a C^{-1 / 2}$, we show how the sixth-order system reduces to either a fifth- or a fourth-order system, enabling us to gain significant insight into the physical 


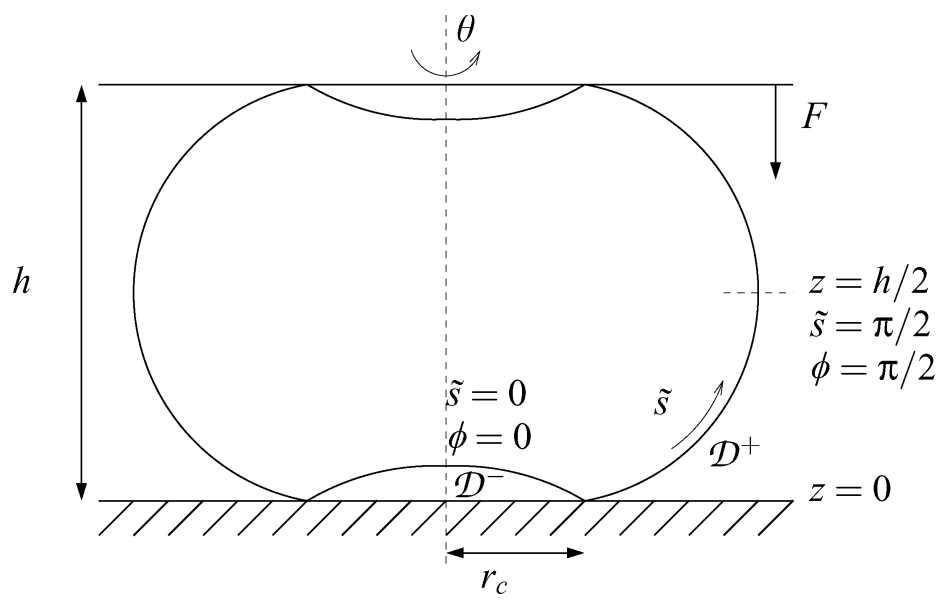

FIGURE 1. Schematic of an axisymmetric vesicle buckled between two plates.

behaviour, revealing the scaling properties of the underlying bifurcation structure, and providing predictions of force-displacement relations that can be tested experimentally.

In Section 2 we outline the derivation of the model used by Preston et al. (2008) for the vesicle's mechanics, discuss the constitutive assumptions and present some numerical results. In Section 3 we investigate the asymptotic behaviour of the vesicle as the inter-plate distance $h$ varies when $C$ is large. For mathematical convenience, we use the arc-length between the revolution axis and the contact point $\tilde{s}_{c}$ (see Figure 1) as the control parameter (instead of $h$ ). We identify four different asymptotic regions in the $\left(C, \tilde{s}_{c}\right)$-parameter space (see Figure 7 below) and use matched asymptotic analysis to derive, to leading order, the shape of the vesicle, the stress resultants, the inter-plate distance and the force exerted by the plates on the vesicle. The buckling instability is addressed in Section 3.4. Our findings are summarised and their physical significance is discussed in Section 4.

\section{Model formulation}

We consider an axisymmetric vesicle under compression between two parallel plates a distance $h$ apart (see Figure 1). The model formulation follows closely that in Preston et al. (2008). Friction between the vesicle and the plates is neglected.

A point on the vesicle is identified by the azimuthal angle $\theta$ and the arc-length $s$ along a meridian, measured from the lower pole (see Figure 2). For an axisymmetric vesicle, the shape is entirely determined by the two functions $r(s)$ and $z(s)$ that describe the distance to the axis of rotation and the lower plate, respectively. Let $\kappa_{\theta}$ and $\kappa_{\phi}$ be the principal curvatures in the azimuthal and meridional directions respectively and $\phi$ be the angle made by the normal to the vesicle with the vertical axis. The variables $r, z, \kappa_{\theta}, \kappa_{\phi}$ and $\phi$ are coupled through the geometrical relations

$$
\kappa_{\theta}=\sin \phi / r, \quad \phi_{s}=\kappa_{\phi}, \quad r_{s}=\cos \phi, \quad z_{s}=\sin \phi,
$$

where the subscript $s$ denotes a derivative with respect to $s$. 


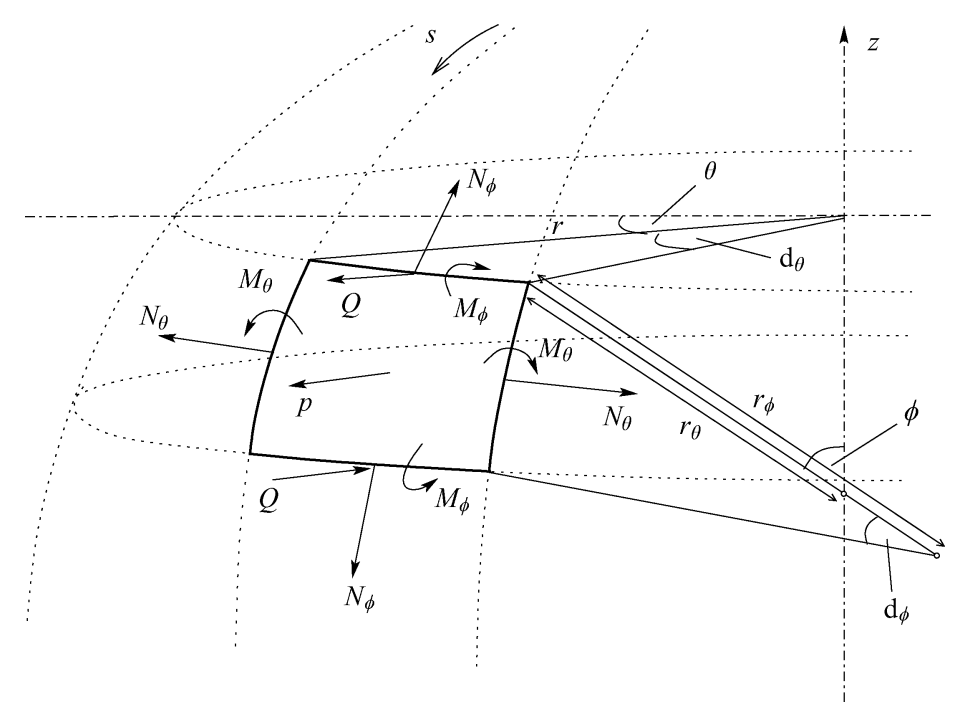

FIGURE 2. Infinitesimal vesicle element.

Figure 2 shows an infinitesimal vesicle element, acted upon by in-plane tensile stress resultants $N_{\theta}$ and $N_{\phi}$, an out-of-plane stress resultant $Q$, in-plane bending stress resultants $M_{\theta}$ and $M_{\phi}$, and a transmural pressure $p$ (defined as the pressure difference between the interior and the exterior of the vesicle). The force- and moment-balance equations on the vesicle element are

$$
\begin{aligned}
\left(N_{\phi} r\right)_{s}-N_{\theta} \cos \phi-Q \kappa_{\phi} r & =0, \\
N_{\phi} \kappa_{\phi}+N_{\theta} \kappa_{\theta}+\frac{1}{r}(Q r)_{s} & =p, \\
\left(M_{\phi} r\right)_{S}-M_{\theta} \cos \phi+Q r & =0 .
\end{aligned}
$$

Equations (2.1) and (2.2) yield seven equations for the ten unknowns $N_{\phi}, N_{\theta}, M_{\phi}, M_{\theta}$, $Q, \kappa_{\phi}, \kappa_{\theta}, r, z$ and $\phi$. The problem is closed by specifying three constitutive equations.

\subsection{Constitutive relations}

Defining the reference shape of the undeformed vesicle by $\tilde{\theta}, \tilde{r}(\tilde{s})$ and $\tilde{z}(\tilde{s})$, where $\tilde{\theta}$ and $\tilde{s}$ are the azimuthal angle and the arc-length around a meridian in the reference configuration respectively, the principal stretches $\lambda_{\phi}$ and $\lambda_{\theta}$ (along the meridians and the parallels, respectively) can be written as $\lambda_{\phi}=\mathrm{d} \tilde{s} / \mathrm{d} s$ and $\lambda_{\theta}=r \mathrm{~d} \theta / \tilde{r} \mathrm{~d} \tilde{\theta}$. We assume that the local surface area of the vesicle is constant (a usual assumption for lipid-bilayer membranes), so that the principal stretches satisfy

$$
\lambda_{\phi}=\frac{1}{\lambda_{\theta}} \equiv \frac{1}{\lambda}
$$


For an axisymmetric deformation (whereby $\mathrm{d} \theta / \mathrm{d} \tilde{\theta}=1$ ) in which the undeformed vesicle is a sphere of radius $a$ (so that in dimensionless terms $\tilde{r}=\sin \tilde{s}$ ) we therefore have that

$$
\lambda=\frac{\mathrm{d} \tilde{s}}{\mathrm{~d} s}=\frac{r}{\sin \tilde{s}}
$$

which provides two extra equations for the two additional variables $\lambda$ and $\tilde{s}$.

Following Pamplona \& Calladine (1993), we assume that the principal tensions and stretches are related by

$$
N_{\phi}-N_{\theta}=H\left(\lambda^{-1}-\lambda\right),
$$

where the shear modulus $H$ measures the resistance to changes in shape in the plane of the vesicle. It is convenient to introduce the isotropic stress resultant $T$, so that (2.5) becomes

$$
N_{\phi}=T+H / \lambda \text { and } N_{\theta}=T+H \lambda .
$$

Following Evans \& Skalak (1980), the bending moments are assumed to be isotropic and proportional to the mean curvature of the surface, which is assumed stress-free in its flat state:

$$
M_{\phi}=M_{\theta}=B\left(\kappa_{\phi}+\kappa_{\theta}\right),
$$

where $B$ is the vesicle's resistance to bending.

The present constitutive model separates bending (2.7) and stretching (2.6) effects, which is a reasonable approximation for a flat element. In contrast, Pamplona et al. (2005) propose a model that includes terms that couple principal tensions and bending moments, accounting also for non-zero spontaneous curvature of the vesicle. To allow for a direct comparison with Preston et al. (2008), these terms have not been included in the present model; indeed, in the parameter regimes of most interest here, involving relatively shallow (but still non-linear) deformations of the vesicle, these terms appear at orders beyond those retained in our analysis, as explained in Appendix A.

\subsection{Non-dimensionalisation}

We non-dimensionalise lengths on the typical length scale of the undeformed vesicle (its radius $a$ ) and forces on $B / a$, changing the variables according to

$$
\begin{aligned}
(r, z, s) & \rightarrow(r, z, s) a, \\
\left(\kappa_{\phi}, \kappa_{\theta}\right) & \rightarrow\left(\kappa_{\phi}, \kappa_{\theta}\right) / a, \\
\left(N_{\phi}, N_{\theta}, T\right) & \rightarrow\left(N_{\phi}, N_{\theta}, T\right) H, \\
Q & \rightarrow Q B / a^{2}, \\
\left(M_{\phi}, M_{\theta}\right) & \rightarrow\left(M_{\phi}, M_{\theta}\right) B / a, \\
p & \rightarrow p B / a^{3}, \\
\lambda & \rightarrow \lambda .
\end{aligned}
$$


After non-dimensionalisation, the material properties of the vesicle are fully encapsulated by the single dimensionless parameter

$$
C=H a^{2} / B
$$

that measures the ratio of the vesicle's resistance to in-plane shearing compared to its resistance to bending. The only geometric parameter is the plate spacing $h \rightarrow h a$.

The non-dimensional equations in Preston et al. (2008) can be obtained from our formulation by replacing our variables $\left(N_{\phi}, N_{\theta}, T\right)$ by $\left(N_{\phi}, N_{\theta}, T\right) / C$.

\subsection{Governing equations}

Equations (2.1), (2.2), (2.5), (2.6) and (2.7) can be written, in dimensionless form, as the system of ordinary differential equations (ODEs):

$$
\begin{aligned}
r_{s} & =\cos \phi, \\
z_{s} & =\sin \phi, \\
\phi_{s} & =\kappa, \\
\kappa_{s} & =-Q+\sin \phi \cos \phi / r^{2}-\kappa \cos \phi / r, \\
Q_{s} & =p-C(T+1 / \lambda) \kappa-C(T+\lambda) \sin \phi / r-Q \cos \phi / r, \\
C T_{s} & =-C(1 / \lambda)_{s}+C(\lambda-1 / \lambda) \cos \phi / r+\kappa Q,
\end{aligned}
$$

with $\kappa \equiv \kappa_{\phi}$ and replacing $\kappa_{\theta}$ by $\sin \phi / r$ as in (2.1). Using (2.4), (2.10) can be written in Lagrangian coordinates (in terms of the independent variable $\tilde{s}$ ) as a system of seven equations for seven unknowns:

$$
\begin{aligned}
r_{\tilde{s}} & =\cos \phi / \lambda, \\
z_{\tilde{s}} & =\sin \phi / \lambda, \\
\phi_{\tilde{s}} & =\kappa / \lambda, \\
\kappa_{\tilde{s}} & =\left[-Q+\sin \phi \cos \phi / r^{2}-\kappa \cos \phi / r\right] / \lambda, \\
Q_{\tilde{s}} & =[p-C(T+1 / \lambda) \kappa-C(T+\lambda) \sin \phi / r-Q \cos \phi / r] / \lambda, \\
C T_{\tilde{s}} & =\left[C \lambda_{\tilde{s}} / \lambda+C(\lambda-1 / \lambda) \cos \phi / r+\kappa Q\right] / \lambda, \\
\lambda & =r / \sin \tilde{s} .
\end{aligned}
$$

The value of the pressure $p$ depends on the model chosen for the vesicle. If the vesicle is assumed to be impermeable and the fluid encapsulated inside incompressible, $p$ must be determined through a volume constraint. In contrast, if the vesicle is porous, then the pressure difference $p$ between the interior and the exterior of the vesicle is zero. Here we assume that the normal stress exerted on the vesicle is zero everywhere except where the vesicle is in contact with the plates.

In any plane for which $\theta=$ Constant, the contact between the vesicle and the bottom plate (say) occurs either along a flat portion of the meridian defined by $0 \leqslant \tilde{s} \leqslant \tilde{s}_{c}$, or at one point on the meridian (when the vesicle buckles) located at $\tilde{s}=\tilde{s}_{c}$. In both cases, it 
has been shown that the pressure is non-zero only at the free boundary $\tilde{s}=\tilde{s}_{c}$ (Preston et al., 2008) (or equivalently $r \equiv r_{c}$ ), so that we can write

$$
p=-F \frac{\delta\left(\tilde{s}-\tilde{s}_{c}\right)}{2 \pi r_{c}}
$$

where $F$ is an undetermined constant representing the force exerted by the plates (see Figure 1) and distributed around the circle of radius $r_{c}$. The corresponding dimensional force is $B F / a$. We wish to compute $F$ and the vesicle shape as a function of the material and geometric parameters $C$ and $h$.

\subsection{Boundary conditions}

Since (2.11e) is undefined at $\tilde{s}=\tilde{s}_{c}$, it is necessary to solve (2.11) in two sub-domains (Figure 1), $\mathscr{D}^{-}\left(0<\tilde{s}<\tilde{s}_{c}\right)$ and $\mathscr{D}^{+}\left(\tilde{s}_{c}<\tilde{s} \leqslant \pi / 2\right)$, with continuity conditions at $\tilde{s}=\tilde{s}_{c}$. Dependent variables defined in $\mathscr{D}^{-}$or $\mathscr{D}^{+}$are labelled with a superscript $(-)$or $(+)$, respectively. Equations with no superscripts are valid both in $\mathscr{D}^{-}$and $\mathscr{D}^{+}$.

At $\tilde{s}=0,(2.11)$ is singular so the boundary conditions are enforced via the asymptotic expansion

$$
\left.\begin{array}{l}
r^{-} \sim \tilde{s}, \quad \kappa^{-} \sim A_{1}, \quad \phi^{-} \sim A_{1} \tilde{s}, \\
Q^{-} \sim A_{2} \tilde{s}, \quad T^{-} \sim-A_{2} / C A_{1}-1
\end{array}\right\} \text { for } \tilde{s} \ll 1,
$$

where $A_{1}$ and $A_{2}$ are undetermined constants. Note that these conditions are not appropriate when a point-force is applied at the poles, a case we are not considering here.

The other boundary conditions for (2.11) are

$$
\begin{aligned}
& \begin{array}{l}
\phi^{+}=\phi^{-}=0, \quad z^{+}=z^{-}=0 \\
\left(r^{+}, \kappa^{+}, T^{+}\right)=\left(r^{-}, \kappa^{+}, T^{-}\right),
\end{array} \\
& \left.\phi^{+}=\pi / 2, \quad Q^{+}=0, \quad Q^{-}=h / 2\right\} \text { at the equator } \tilde{s}=\pi / 2 .
\end{aligned}
$$

Thus (2.13a) and (2.13c) represent eight boundary conditions with two unknown parameters $\left(A_{1}\right.$ and $\left.A_{2}\right)$ and one prescribed parameter $(h)$, as might be expected for the sixth-order system (2.11), while (2.13b) represents eight jump conditions with two additional unknown parameters $F$ and $\tilde{s}_{c}$, again as might be expected for a sixth-order system of equations.

The boundary conditions $(2.13 a-c)$, a detailed derivation of which is given in Preston et al. (2008), are valid whether or not the vesicle is buckled; in the unbuckled case, all the dependent variables but the tension $T^{-}$are zero inside the contact disk at the poles, leading to $A_{1}=A_{2}=0$ in $(2.13 a)$.

\subsection{Numerical solutions}

Figures 3, 4 and 5 show solutions of the full system (2.11) for large values of $C$, as obtained numerically following Preston et al. (2008) (the figures also contain the results from our asymptotic analysis, which will be discussed in Section 3). The method employed consists in solving the sixth-order system (2.11) separately in $\mathscr{D}^{+}$and $\mathscr{D}^{-}$, with boundary 
(a)

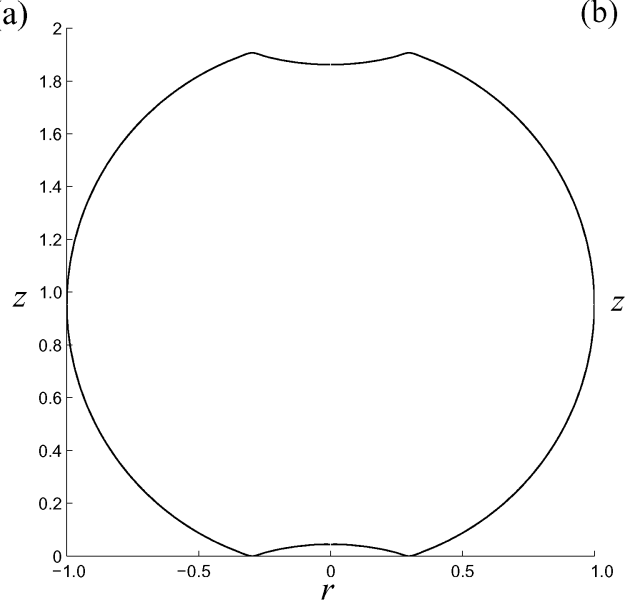

(b)

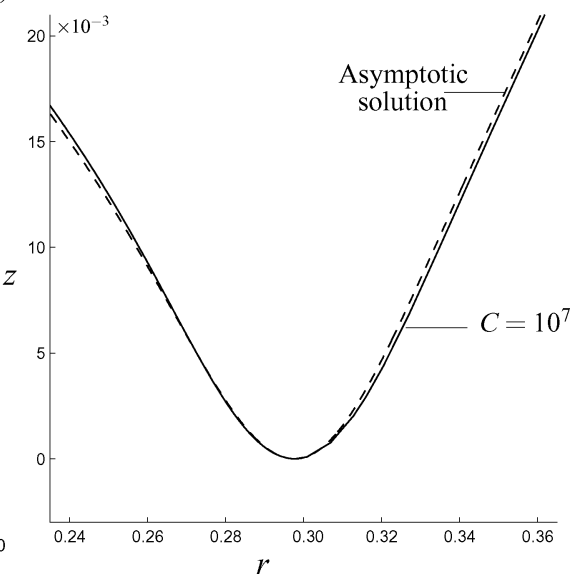

FIGURE 3. Shape of the buckled vesicle for $\tilde{s}_{c}=0.3$ and $C=10^{7}$. The numerical solution of the full system (2.11)-(2.13) (solid) and the composite solution $\tilde{z}_{\text {comp }}$ (3.33) from leading-order asymptotics in the outer and in the inner regions (dashed) are shown for (a) the whole vesicle (the two curves are graphically indistinguishable); (b) the inner region near one of the contact points.

conditions at each end as described by (2.13), using MATLAB's boundary-value-problem solver bvp4c. The problem is well posed provided $F$ or $h$ is prescribed, but the solution is not always unique as regimes of bistability have been identified whereby the vesicle can be buckled or unbuckled (Murray \& Wright, 1961; Preston et al., 2008).

For $C \gg 1$, the general shape of the buckled vesicle can be described approximately by sectors of spheres (Figure 3), with large variations of the slope $\phi$ occurring only in the vicinity of the contact point. This region is also where most of the stresses are confined, as shown by the different scalings used between Figures 4 (showing how variables evolve over the domain $0 \leqslant \tilde{s} \leqslant \pi / 2$ ) and 5 (showing variables in the neighbourhood of the contact point). As expected for a vesicle compressed between two plates, the meridional stress component $N_{\phi}$ is predominantly negative, implying compression (Figures 4(c) and 5(c)). The azimuthal stress component $N_{\theta}$ changes sign at the contact point, so that the vesicle is under azimuthal compression on the inside of the contact line $\left(\tilde{s}<\tilde{s}_{c}\right)$ and is predominantly under extension $\left(N_{\theta}>0\right)$ elsewhere (Figure 5(d)).

With appropriate scalings in powers of $C^{1 / 4}$, all dependent variables in (2.11) are found to collapse as $C \rightarrow \infty$ within an inner region of width $\mathcal{O}\left(C^{-1 / 4}\right)$ around the free boundary $\tilde{s}=\tilde{s}_{c}$ (see Figure 5). With different scalings, the dependent variables also collapse outside the inner region as $C \rightarrow \infty$ (see Figure 4). This motivates an asymptotic analysis for $C \gg 1$, considering two outer regions $\mathscr{D}_{\text {out }}^{-}$and $\mathscr{D}_{\text {out }}^{+}$, connected by the inner region $\mathscr{D}_{\text {in }}$ near $\tilde{s}=\tilde{s}_{c}$.

Figure 6 shows the force-displacement relationship $F$ versus $h$ computed numerically following Preston et al. (2008). The figure illustrates the sub-critical bifurcation (using the plate separation $h$ as the control parameter) between unbuckled and buckled configurations. In the asymptotic analysis that follows (Section 3), we explore in detail the proposed scaling behaviour of the force for post-buckled solutions, $F \propto C^{1 / 4}(1-h / 2)^{1 / 2}$ 

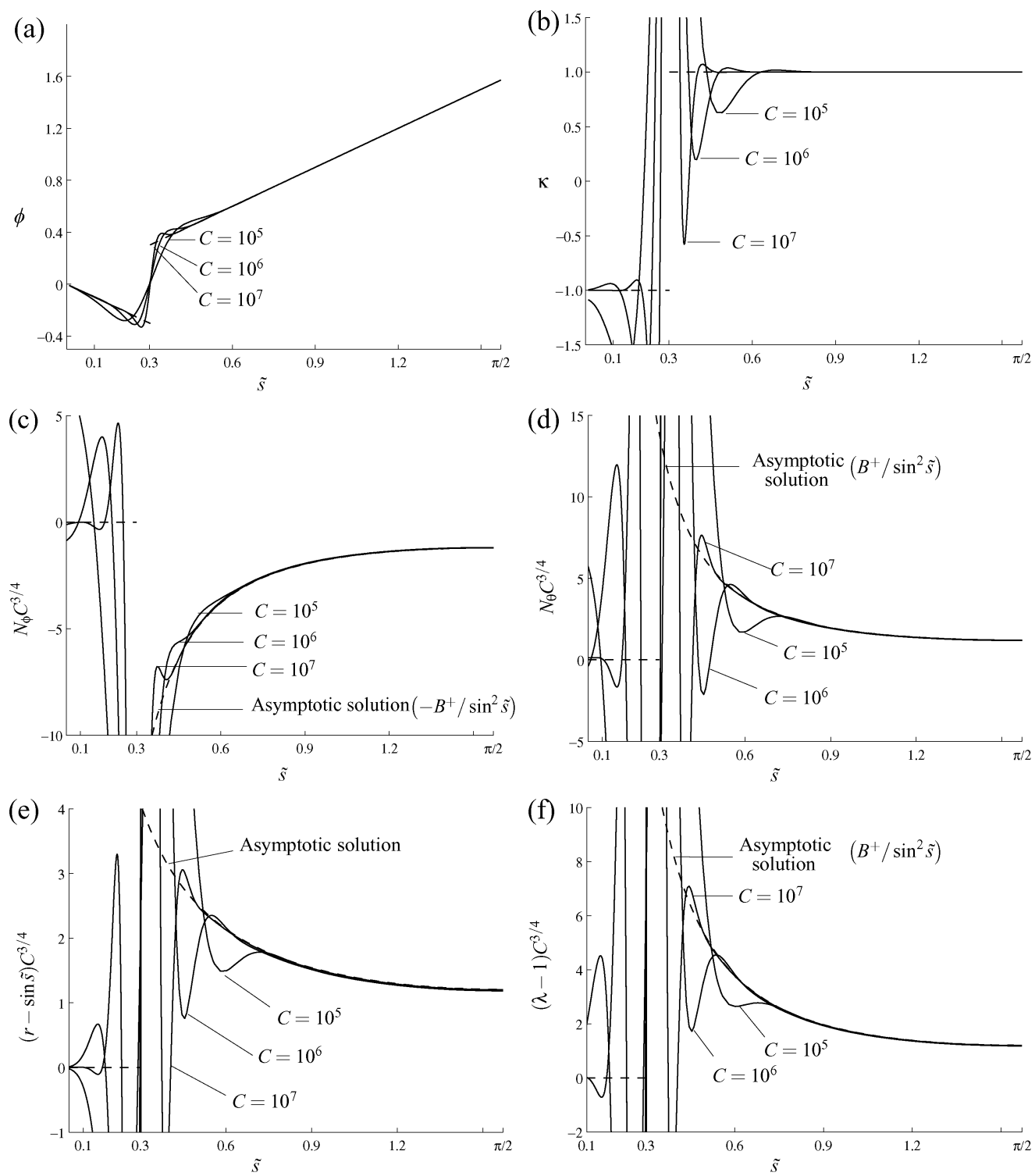

FIGURE 4. Numerical solutions of the full system (2.11)-(2.13) (solid) for $\tilde{s}_{c}=0.3$ and $C=$ $\left[10^{5}, 10^{6}, 10^{7}\right]$ and leading-order outer asymptotic solution (3.6), (3.12) and (3.14) (dashed), both re-scaled to be $\mathcal{O}(1)$ in the outer regions. (a) $\phi$; (b) $\kappa$; (c) $N_{\phi} C^{3 / 4}$; (d) $N_{\theta} C^{3 / 4}$; (e) $(r-\sin \tilde{s}) C^{3 / 4}$; (f) $C^{3 / 4}(\lambda-1)$ versus $\tilde{s}$. The vertical sections of curves in (b)-(f) are resolved under inner-region scalings shown in Figure 5.

as $C \rightarrow \infty$, as identified in Komura et al. (2005) and Preston et al. (2008), and the onset of the buckling instability for $(1-h / 2)=\mathcal{O}\left(C^{-1 / 2}\right)$. We summarise in Section 4 the findings of the asymptotic analysis, to which readers who are less interested in technical details may turn directly. 

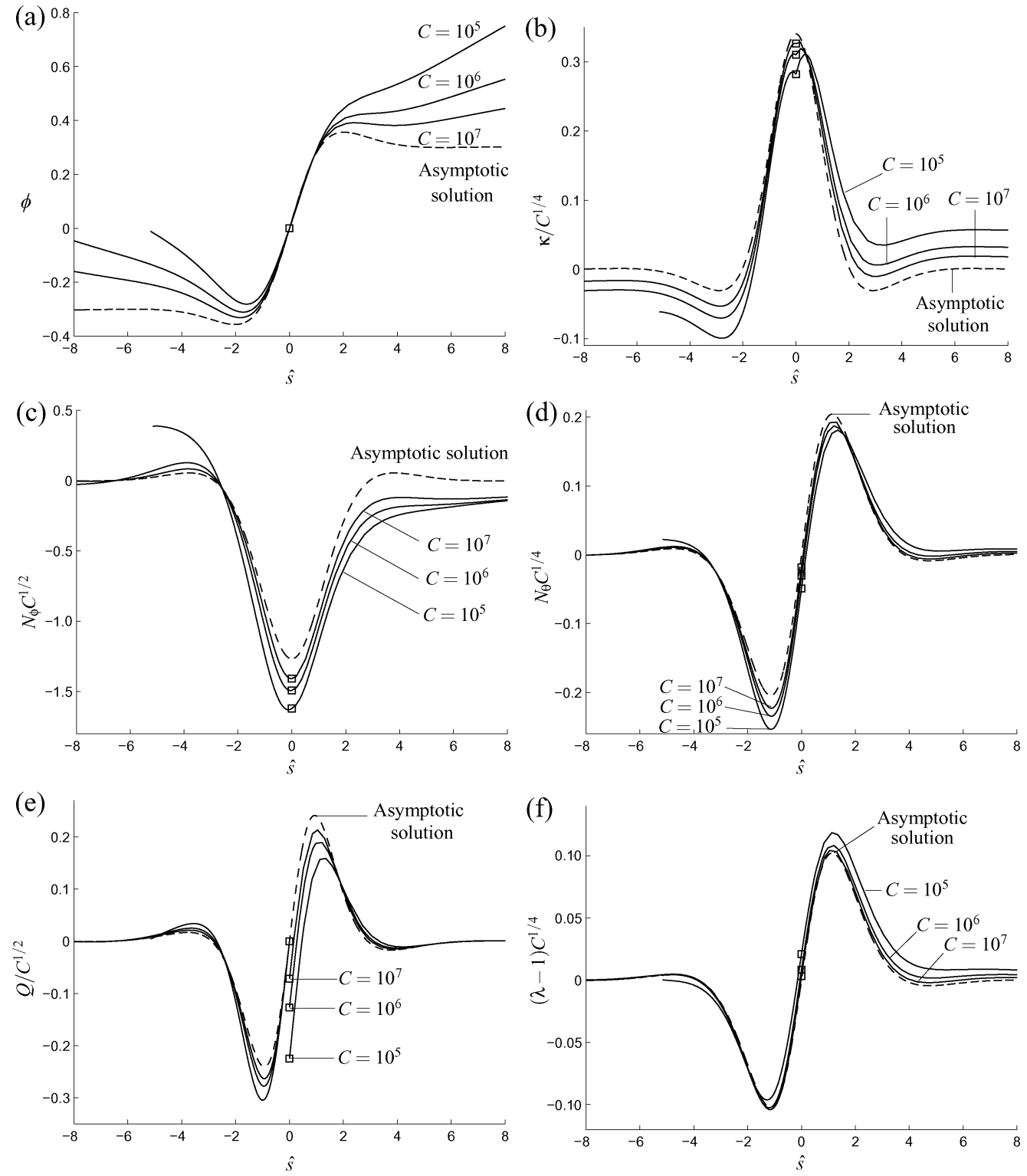

FIGURE 5. Numerical solutions of the full system (2.11)-(2.13) (solid) plotted in inner coordinates for $\tilde{s}_{c}=0.3$ and $C=\left[10^{5}, 10^{6}, 10^{7}\right]$ and the leading-order asymptotic solution (3.29-3.30) (dashed). (a) $\phi$; (b) $\kappa / C^{1 / 4}$; (c) $N_{\phi} C^{1 / 2}$; (d) $N_{\theta} C^{1 / 4}$; (e) $Q / C^{1 / 2}$; (f) $C^{1 / 4}(\lambda-1)$ versus $\hat{s}=C^{1 / 4}\left(\tilde{s}-\tilde{s}_{c}\right.$ ). Squares show the contact point.

\section{Asymptotic solution for $C \gg 1$}

It is convenient to parametrise solutions using $\tilde{S}_{c}$ instead of $h$, so we assume that $\tilde{S}_{c}$ is fixed and expand $h$ as follows:

$$
h=h_{0}+C^{-1 / 4} h_{1}+C^{-1 / 2} h_{2} \ldots
$$




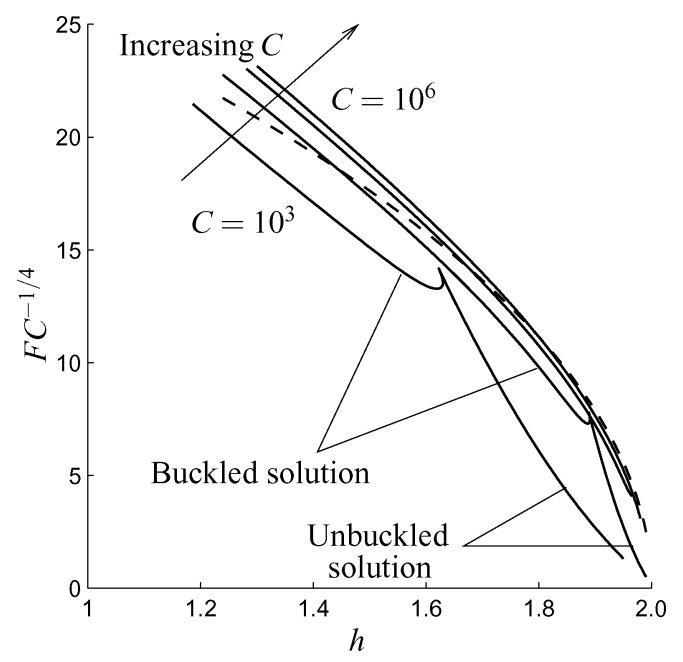

FIGURE 6. Solid lines show the scaled force $F$, versus inter-plate distance for $C=\left[10^{3}, 10^{4}, 10^{5}, 10^{6}\right]$. The dashed line corresponds to the leading-order asymptotic prediction for region II (3.44), which is valid for $h$ close to 2 , in the post-buckled state.

Because the problem has two independent parameters (now $\tilde{s}_{c}$ and $C$ ), we must be careful in identifying precisely the limiting cases that arise for $C \gg 1$. Various regimes are considered, depending on the distance of the contact ring from the axis. As illustrated in Figure 7, we will consider the following regimes that arise under different forcings and plate spacings: I, $C^{-1 / 4} \ll \tilde{s}_{c} \sim 1$ (where ' $\sim 1$ ' means $\tilde{s}_{c}=\mathcal{O}(1)$ ); II, $C^{-1 / 4} \ll \tilde{s}_{c} \ll 1$; III, $C^{-1 / 4} \sim \tilde{S}_{c} \ll 1$; and IV, $C^{-1 / 2} \ll \tilde{S}_{c} \ll C^{-1 / 4} \ll 1$. As detailed below, the distinguished limits I and III represent a buckled vesicle with $\mathcal{O}(1)$ deformations and a weakly deformed vesicle near the buckling instability respectively. The behaviour of the unbuckled solution for asymptotically small deformations is addressed in case IV. We focus initially on case II, which concerns a vesicle that is strongly buckled over a small region, since it is physically relevant and, in the context of this study, analytically tractable. Here an approximate solution of the system (2.11) with (2.13) is constructed by asymptotic expansions in both $\mathscr{D}_{\text {out }}^{-}$and $\mathscr{D}_{\text {out }}^{+}$with matching conditions through a boundary layer $\mathscr{D}_{\text {in }}$.

We present the outer solution in Section 3.1; we discuss the inner problem in case I in Section 3.2 and show how it simplifies in case II in Section 3.3; in Section 3.4 we show how to recover the bifurcation structure in case III; and in Section 3.5 we address case IV.

\subsection{Outer region}

In the outer regions we write:

$$
\begin{aligned}
r & =\bar{r}_{0}+C^{-3 / 4} \bar{r}_{1}+\cdots, \quad T=\bar{T}_{0}+C^{-3 / 4} \bar{T}_{1}+\cdots, \quad \lambda=\bar{\lambda}_{0}+C^{-3 / 4} \bar{\lambda}_{1}+\cdots \\
\phi & =\bar{\phi}_{0}+C^{-3 / 4} \bar{\phi}_{1}+\cdots, \quad \kappa=\bar{\kappa}_{0}+C^{-3 / 4} \bar{\kappa}_{1}+\cdots, \quad Q=\bar{Q}_{0}+C^{-3 / 4} \bar{Q}_{1}+\cdots \\
z & =\bar{z}_{0}+C^{-1 / 4} \bar{z}_{1}+C^{-1 / 2} \bar{z}_{2}+C^{-3 / 4} \bar{z}_{3}+\cdots
\end{aligned}
$$


(a)

(c)

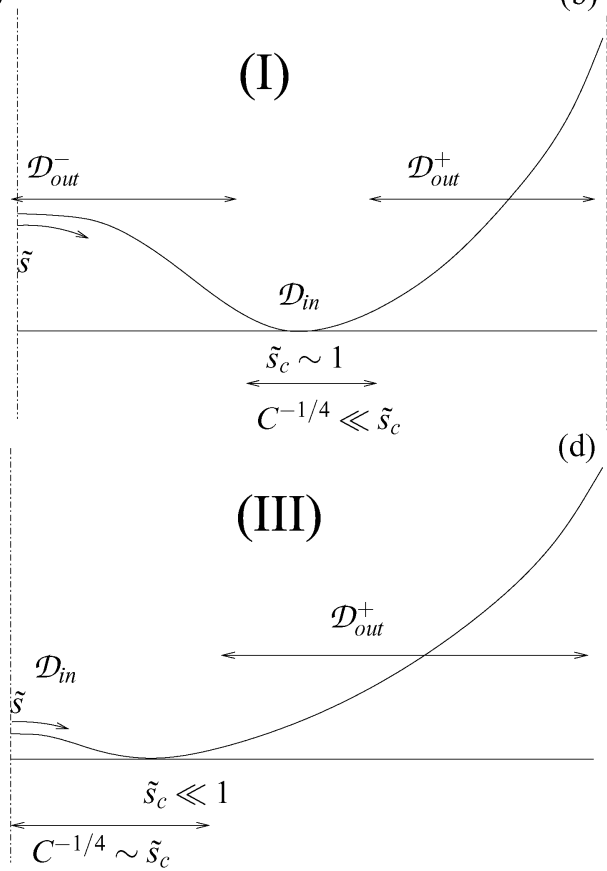

(b)
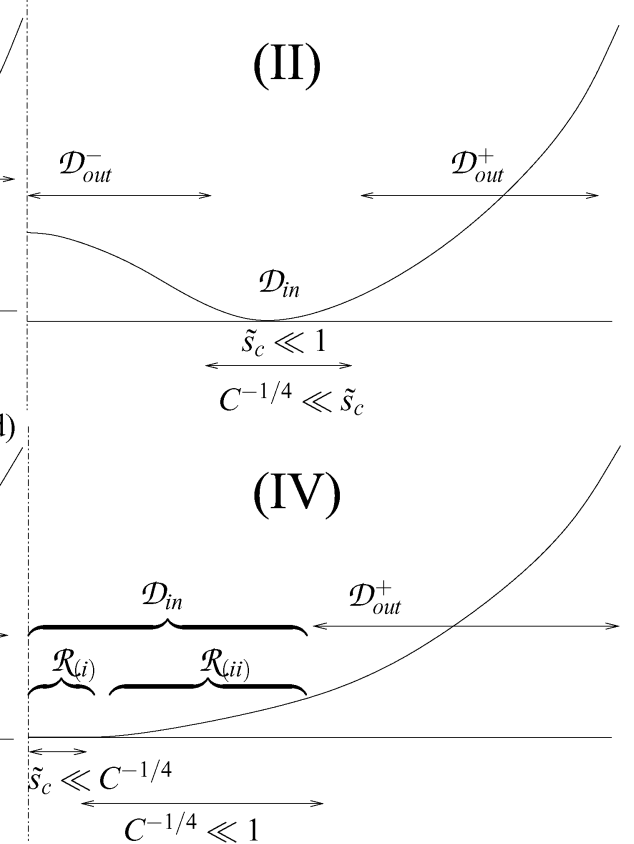

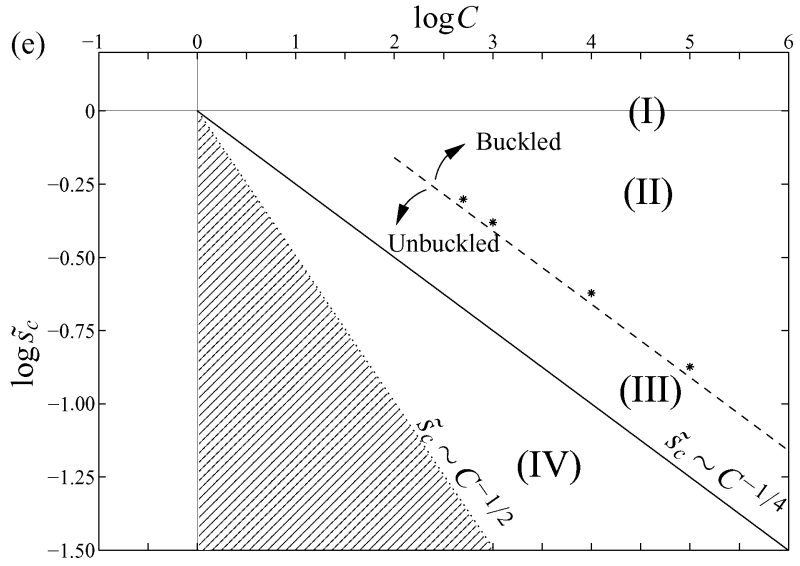

FIGURE 7. (a)-(d) Sketches representing the asymptotic solution structure in regions I-IV, respectively. (e) Parameter space showing regions I, II, III and IV. The dashed line (with slope -1/4) represents the buckling instability, as predicted by the leading-order analysis (3.59). The asterisks are data points for the onset of the buckling instability for $C=\left[500,10^{3}, 10^{4}, 10^{5}\right]$ as computed numerically by Preston et al. (2008) (the transcritical and saddle-node bifurcation points are here graphically indistinguishable). In the hashed area, the shell theory assumptions are not likely to be satisfied as $\tilde{s}_{c}$ will not be larger than the shell thickness. 
where bars denote functions of $\tilde{s}$. Recall that expressions written without superscripts are valid in both $\mathscr{D}_{\text {out }}^{-}$and $\mathscr{D}_{\text {out }}^{+}$.

\subsubsection{Leading order}

To leading order for $C \gg 1,(2.11 a-g)$ becomes the fifth-order system

$$
\begin{aligned}
\bar{r}_{0 \tilde{s}} & =\cos \bar{\phi}_{0} / \bar{\lambda}_{0}, \\
\bar{z}_{0 \tilde{s}} & =\sin \bar{\phi}_{0} / \bar{\lambda}_{0}, \\
\bar{\phi}_{0 \tilde{s}} & =\bar{\kappa}_{0} / \bar{\lambda}_{0}, \\
\bar{\kappa}_{0 \tilde{s}} & =\left[-\bar{Q}_{0}+\sin \bar{\phi}_{0} \cos \bar{\phi}_{0} / \bar{r}_{0}^{2}-\bar{\kappa}_{0} \cos \bar{\phi}_{0} / \bar{r}_{0}\right] / \bar{\lambda}_{0}, \\
0 & =-\left(\bar{T}_{0}+1 / \bar{\lambda}_{0}\right) \bar{\kappa}_{0}-\left(\bar{T}_{0}+\bar{\lambda}_{0}\right) \sin \bar{\phi}_{0} / \bar{r}_{0}, \\
\bar{\lambda}_{0} \bar{T}_{0 \tilde{s}} & =\bar{\lambda}_{0 \tilde{s}} / \bar{\lambda}_{0}+\left(\bar{\lambda}_{0}-1 / \bar{\lambda}_{0}\right) \cos \bar{\phi}_{0} / \bar{r}_{0}, \\
\bar{\lambda}_{0} & =\bar{r}_{0} / \sin \tilde{s},
\end{aligned}
$$

in both $\mathscr{D}_{\text {out }}^{+}$and $\mathscr{D}_{\text {out }}^{-}$. The boundary conditions satisfied by the solution in $\mathscr{D}_{\text {out }}^{-}$and $\mathscr{D}_{\text {out }}^{+}$ are respectively

$$
\left.\begin{array}{l}
\bar{r}_{0}^{-} \sim \tilde{s}, \quad \bar{\kappa}_{0}^{-} \sim A_{1}, \\
\bar{\phi}_{0}^{-} \sim A_{1} \tilde{s}, \quad \text { and } \quad \bar{Q}_{0}^{-} \sim A_{2} \tilde{s}
\end{array}\right\} \text { for } \quad \tilde{s} \ll 1,
$$

and

$$
\bar{\phi}_{0}^{+}=\pi / 2, \quad \bar{Q}_{0}^{+}=0, \text { and } \bar{z}_{0}^{+}=h_{0} / 2 \text { at } \quad \tilde{s}=\pi / 2,
$$

where $A_{1}, A_{2}$ and $h_{0}$ are undetermined parameters which depend on $\tilde{s}_{c}$. In addition, we will show, by considering an inner region about the contact point, that the following conditions hold:

$$
\left(\bar{r}_{0}^{+}, \bar{T}_{0}^{+}\right)=\left(\bar{r}_{0}^{-}, \bar{T}_{0}^{-}\right), \quad \text { and } \quad \bar{z}_{0}^{+}=\bar{z}_{0}^{-}=0, \text { as } \quad \tilde{s} \rightarrow \tilde{s}_{c} \pm
$$

Equations $(3.4 a-b)$ give seven boundary conditions at $\tilde{s}=0, \pi / 2$ with two unknown parameters; $(3.4 c)$ gives four conditions at the contact point $\tilde{s}_{c}$ that are derived from matching conditions with the inner region (see below). In particular, we find below that the outer limit of $z$ in the inner region is $\mathcal{O}\left(C^{-1 / 4}\right)$ or smaller (depending on the size of $\tilde{s}_{c}$ ), leading to the zero boundary condition (3.4c) imposed on $\bar{z}_{0}$ as $\tilde{s} \rightarrow \tilde{s}_{c}$.

Equations $(3.3 e-f)$ are satisfied by

$$
\bar{\lambda}_{0}=1, \quad \text { and } \quad \bar{T}_{0}=-1,
$$

so that $N_{\phi}=0$ and $N_{\theta}=0$ to this order. Hence $(3.3 g)$ implies $\bar{r}_{0}=\sin \tilde{s}$ and $(3.3 a-b)$ 
yield

$$
\begin{aligned}
& \bar{z}_{0}^{ \pm}= \pm \begin{cases}\cos \tilde{s}_{c}-\cos \tilde{s}, & \text { if } \tilde{s}_{c}=\mathcal{O}(1), \\
1-\cos \tilde{s}, & \text { if } \tilde{s}_{c}=\mathcal{O}\left(C^{-1 / 4}\right),\end{cases} \\
& \bar{\phi}_{0}^{ \pm}= \pm \tilde{s} .
\end{aligned}
$$

As expected, the vesicle lies along sectors of spheres, with the buckling point at $\bar{r}_{0}=\sin \tilde{s}_{c}$, $\bar{z}_{0}=0$ and a region of reversed curvature (as compared to the initial configuration) in $\mathscr{D}_{\text {out }}^{-}$(see Figure 3(a)). The distance between the plates is, to leading order,

$$
h_{0}=2 z_{0}(\pi / 2)= \begin{cases}2 \cos \tilde{s}_{c} & \text { if } \tilde{s}_{c}=\mathcal{O}(1), \\ 2 & \text { if } \tilde{s}_{c}=\mathcal{O}\left(C^{-1 / 4}\right) .\end{cases}
$$

Solving for $\kappa$ and $Q$, to leading order, using $(3.3 c-d)$ yields

$$
\bar{Q}_{0}^{ \pm}=0, \quad \text { and } \quad \bar{\kappa}_{0}^{ \pm}= \pm 1 .
$$

The outer problem does not capture the jump in the out-of-plane stress component (Figure 5e): the jump condition (2.13b) is satisfied within a boundary layer near the contact point, as could be anticipated from the fact that the derivative $Q_{\tilde{s}}$ does not appear in the leading-order outer problem (3.3).

To leading order, the outer variables $\bar{r}, \bar{\lambda}$ and $\bar{T}$ are continuous across $\tilde{s}=\tilde{s}_{c}$, but $\bar{\phi}, \bar{z}_{\tilde{s}}$ and $\bar{\kappa}$ are not. We expect these quantities to be regularised within the inner region.

The next two orders in the expansion of (2.11) and (2.13) are trivial and yield $\bar{z}_{1 s}=$ $\bar{z}_{2 s}=0$. Therefore, $\bar{z}_{1}$ and $\bar{z}_{2}$ are constants in $\mathscr{D}_{\text {out }}^{+}$and $\mathscr{D}_{\text {out }}^{-}$. They are determined below through matching to the inner region $\mathscr{D}_{i n}$. However, to formulate well-posed matching conditions between $\mathscr{D}_{\text {out }}^{ \pm}$and $\mathscr{D}_{\text {in }}$, we need to proceed one step further in the expansion of (2.11) and (2.13).

\subsubsection{Next order}

To next order in the large- $C$ expansion, (3.2) is given by the system

$$
\begin{aligned}
\bar{r}_{1 s} & =-\bar{\phi}_{1} \sin \bar{\phi}_{0}-\bar{\lambda}_{1} \cos \bar{\phi}_{0}, \\
\bar{z}_{3 s} & =\bar{\phi}_{1} \cos \bar{\phi}_{0}-\bar{\lambda}_{1} \sin \bar{\phi}_{0}, \\
\bar{\phi}_{1 s} & =\bar{\kappa}_{1}-\bar{\lambda}_{1} \bar{\kappa}_{0}, \\
\bar{\kappa}_{1 s} & =-\bar{Q}_{1}+\frac{\cos \bar{\phi}_{0}}{\bar{r}_{0}}\left(-\bar{\lambda}_{1}+\bar{\phi}_{1} \cot \tilde{s}-\bar{\kappa}_{1}\right), \\
0 & =-\left(\bar{T}_{1}-\bar{\lambda}_{1}\right) \bar{\kappa}_{0}-\left(\bar{T}_{1}+\bar{\lambda}_{1}\right) \frac{\sin \bar{\phi}_{0}}{\bar{r}_{0}}, \\
\bar{T}_{1 s} & =\bar{\lambda}_{1 s}+2 \bar{\lambda}_{1} \frac{\cos \bar{\phi}_{0}}{\bar{r}_{0}}, \\
\bar{\lambda}_{1} & =\bar{r}_{1} / \sin \tilde{s} .
\end{aligned}
$$


Equation $(3.9 e)$ yields $\bar{T}_{1}^{ \pm}=0\left(\right.$ since $\left.\bar{\kappa}_{0}=\sin \bar{\phi}_{0} / \bar{r}_{0}\right)$, so that $(3.9 f)$ becomes

$$
0=\bar{\lambda}_{1 s}^{ \pm} / \bar{\lambda}_{1}^{ \pm}+2 \cot \tilde{s}
$$

Hence $\bar{\lambda}_{1}^{-}=B^{-} / \sin ^{2} \tilde{s}$, for some $\mathcal{O}(1)$ constant $B^{-}$. Since $\bar{\lambda}_{1}^{-}$must remain bounded as $\tilde{s} \rightarrow 0, B^{-}=0$ necessarily and we have

$$
\bar{T}_{1}^{-}=0, \quad \bar{\lambda}_{1}^{-}=0
$$

Substituting $\bar{\lambda}_{1}^{-}=0$ into $(3.9 g)$ gives $\bar{r}_{1}^{-}=0$, which in turn yields

$$
\bar{\phi}_{1}^{-}=\bar{\kappa}_{1}^{-}=\bar{Q}_{1}^{-}=0
$$

using $(3.9 a-d)$ respectively.

Likewise, in $\mathscr{D}_{\text {out }}^{+}$we have

$$
\bar{T}_{1}^{+}=0, \quad \bar{\lambda}_{1}^{+}=B^{+} / \sin ^{2} \tilde{s}
$$

for some $\mathcal{O}(1)$ constant $B^{+}$(to be determined by matching with the solution in the inner region). Substituting $(3.13 b)$ in $(3.9 g)$ and using $(3.9 a-d)$ gives

$$
\bar{r}_{1}^{+}=B^{+} / \sin \tilde{s}, \quad \bar{\phi}_{1}^{+}=0, \quad \bar{\kappa}_{1}^{+}=B^{+} / \sin ^{2} \tilde{s}, \quad \text { and } \quad \bar{Q}_{1}^{+}=0 .
$$

The scalings in $\mathcal{O}\left(C^{-3 / 4}\right)$ of $\bar{r}_{1}^{+}$and $\bar{\lambda}_{1}^{+}$are confirmed in Figure $4(e-f)$ respectively.

The solutions obtained in $\mathscr{D}_{\text {out }}^{ \pm}$will be used below as matching conditions to determine the solution in the inner region $\mathscr{D}_{\text {in }}$ and $B^{+}$will be determined by a solvability condition in Section 3.3.1 below. A physical interpretation of $B^{+}$is as the leading-order perturbation of the radius of the vesicle at the equator: $\left.r\right|_{\tilde{s}=\pi / 2}=1+B^{+} C^{-3 / 4}$. In the case presented in Figure $4\left(\tilde{s}_{c}=0.3\right)$, a fit from the data for $\bar{\lambda}_{1}^{+}$and $\bar{r}_{1}^{+}$yields $B^{+} \approx-1.2$, which agrees with the predicted value from our analysis below ((3.36), (3.44a)).

Expanding the outer solution as $\tilde{s} \rightarrow \tilde{s}_{c}$ with $\tilde{s}-\tilde{s}_{c}=\mathcal{O}\left(C^{-1 / 4}\right)$ (the scale of the inner variable) to the first few orders, we may express the inner limit of the outer problem as

$$
\begin{aligned}
& r=\sin \tilde{s}_{c}+\mathcal{O}\left(C^{-1 / 4}\right), \\
& T=-1+\mathcal{O}\left(C^{-3 / 2}\right), \\
& \lambda=1+C^{-3 / 4} B^{ \pm} / \sin ^{2} \tilde{s}_{c}+\mathcal{O}\left(B^{ \pm} C^{-1}\right) \\
& \phi= \pm \tilde{s}_{c} \pm\left(\tilde{s}-\tilde{s}_{c}\right)+\mathcal{O}\left(C^{-3 / 2}\right), \\
& \kappa= \pm 1+C^{-3 / 4} B^{ \pm} / \sin ^{2} \tilde{s}_{c}+\mathcal{O}\left(B^{ \pm} C^{-1}\right), \\
& Q=\mathcal{O}\left(C^{-3 / 2}\right),
\end{aligned} \text { as }\left\{\begin{array}{l}
\tilde{s} \rightarrow \tilde{s}_{c} \pm, \text { with } \\
\tilde{s}-\tilde{s}_{c}=\mathcal{O}\left(C^{-1 / 4}\right),
\end{array}\right.
$$

where $B^{-}=0$ and $B^{+}$will be determined in the inner region. The asymptotic behaviour (3.15) and the ordering of the different terms hold regardless of the size of $\tilde{s}_{c}$ (at least in the range of interest $\tilde{s}_{c} \gg C^{-1 / 2}$, as discussed below). Since the leading-order term for $z$ depends on $\tilde{s}_{c}(3.6)$, one has to distinguish the case $\tilde{s}_{c} \gg \mathcal{O}\left(C^{-1 / 4}\right)$, whereby

$$
z=\left[\sin \tilde{s}_{c}\left(\tilde{s}-\tilde{s}_{c}\right)+\bar{z}_{1} C^{-1 / 4}\right]+\left[\frac{1}{2} \cos \tilde{s}_{c}\left(\tilde{s}-\tilde{s}_{c}\right)^{2}+\bar{z}_{2} C^{-1 / 2}\right]+\mathcal{O}\left(C^{-3 / 4}\right),
$$


and $\tilde{s}_{c}=\mathcal{O}\left(C^{-1 / 4}\right)$, for which

$$
z=\bar{z}_{1} C^{-1 / 4}+\left[\frac{1}{2} \tilde{s}_{c}^{2}+\tilde{s}_{c}\left(\tilde{s}-\tilde{s}_{c}\right)+\frac{1}{2}\left(\tilde{s}-\tilde{s}_{c}\right)^{2}+\bar{z}_{2} C^{-1 / 2}\right]+\mathcal{O}\left(C^{-3 / 4}\right)
$$

when $\tilde{s} \rightarrow \tilde{s}_{c}^{ \pm}$with $\tilde{s}-\tilde{s}_{c}=\mathcal{O}\left(C^{-1 / 4}\right)$. The constants $\left(\bar{z}_{1}, \bar{z}_{2}\right)$ will be determined in the inner region, separately for cases II and III.

Proceeding this far in the expansion of the outer problem provides the matching conditions (3.15-3.17) necessary to solve the inner problem (formulated in the next section for four different cases) and derive the leading-order asymptotic behaviour of the vesicle.

\subsection{Inner region, case I (i.e. $C^{-1 / 4} \ll \tilde{S}_{c} \sim 1$ )}

We first consider a strongly buckled vesicle, for which the size of the inner region near the contact line is small compared to the radius $\sin \tilde{s}_{c}$ of the contact line (see Figure 7).

Writing (2.11) in term of the inner variable $\hat{s}$, defined by

$$
\tilde{s}=\tilde{s}_{c}+C^{-1 / 4} \hat{s}
$$

and using the dimensionless version of (2.6) yields the re-scaled equations

$$
\begin{aligned}
r_{\hat{s}} & =C^{-1 / 4} \cos \phi / \lambda \\
z_{\hat{s}} & =C^{-1 / 4} \sin \phi / \lambda \\
\phi_{\hat{s}} & =C^{-1 / 4} \kappa / \lambda \\
\kappa_{\hat{s}} & =C^{-1 / 4}\left[-Q+\sin \phi \cos \phi / r^{2}-\kappa \cos \phi / r\right] / \lambda, \\
Q_{\hat{s}} & =\left[C^{-1 / 4} p-C^{3 / 4} N_{\phi} \kappa-C^{3 / 4}\left(N_{\phi}+\lambda-1 / \lambda\right) \sin \phi / r-C^{-1 / 4} Q \cos \phi / r\right] / \lambda, \\
N_{\phi \hat{s}} & =\left[C^{-1 / 4}(\lambda-1 / \lambda) \cos \phi / r+C^{-5 / 4} \kappa Q\right] / \lambda, \\
\lambda & =r / \sin \left(\tilde{s}_{c}+C^{-1 / 4} \hat{s}\right) .
\end{aligned}
$$

Motivated by numerical simulations (see Figure (5)), we thus write

$$
\begin{aligned}
\tilde{s} & =\tilde{s}_{c}+C^{-1 / 4} \hat{s}, & F & =F_{0} C^{1 / 4}+\cdots, \\
r & =\sin \tilde{s}_{c}+C^{-1 / 4} \hat{r}_{1}+C^{-1 / 2} \hat{r}_{2}+\cdots, & \kappa & =C^{1 / 4} \hat{\kappa}_{1}+\hat{\kappa}_{2}+\cdots, \\
z & =C^{-1 / 4} \hat{z}_{1}+C^{-1 / 2} \hat{z}_{2}+\cdots, & Q & =C^{1 / 2} \hat{Q}_{1}+C^{1 / 4} \hat{Q}_{2}+\cdots, \\
\phi & =\hat{\phi}_{1}+C^{-1 / 4} \hat{\phi}_{2}+\cdots, & T & =-1+\hat{T}_{0} C^{-1 / 4}+\hat{T}_{1} C^{-1 / 2}+\hat{T}_{2} C^{-3 / 4}+\cdots, \\
p & =p_{0} \delta(\hat{s}) C^{1 / 2}+\cdots, & \lambda & =1+\hat{\lambda}_{0} C^{-1 / 4}+\hat{\lambda}_{1} C^{-1 / 2}+\hat{\lambda}_{2} C^{-3 / 4}+\cdots,
\end{aligned}
$$

where hats denote functions of $\hat{s}$. We also define $\hat{N}_{1}=\hat{T}_{1}-\hat{\lambda}_{1}+\hat{\lambda}_{0}^{2}$, such that the 
expansion for the stress $N_{\phi}$ along the meridians becomes

$$
N_{\phi}=\left(\hat{T}_{0}-\hat{\lambda}_{0}\right) C^{-1 / 4}+\hat{N}_{1} C^{-1 / 2}+\cdots
$$

After substitution of (3.19) in (3.18), only the second term in the right-hand side of (3.18e) is $\mathcal{O}(C)$, which gives $\hat{T}_{0}=\hat{\lambda}_{0}$.

Proceeding to $\mathcal{O}\left(C^{3 / 4}\right)$ in $(3.18)$, we have

$$
\begin{aligned}
\hat{r}_{1 \hat{s}} & =\cos \hat{\phi}_{1}, & & \hat{\kappa}_{1 s}=-\hat{Q}_{1}, \\
\hat{z}_{1 \hat{s}} & =\sin \hat{\phi}_{1}, & & \hat{Q}_{1 \hat{s}}=-\hat{N}_{1} \hat{\kappa}_{1}-2 \hat{\lambda}_{0} \sin \hat{\phi}_{1} / \sin \tilde{s}_{c}, \\
\hat{\phi}_{1 \hat{s}} & =\hat{\kappa}_{1}, & & \hat{N}_{1 \hat{s}}=2 \hat{\lambda}_{0} \cos \hat{\phi}_{1} / \sin \tilde{s}_{c}+\hat{\kappa}_{1} \hat{Q}_{1}, \\
\hat{\lambda}_{0} & =\left(\hat{r}_{1}-\hat{s} \cos \tilde{s}_{c}\right) / \sin \tilde{s}_{c} . & &
\end{aligned}
$$

The $\hat{r}_{1}$ and $\hat{z}_{1}$ equations decouple from the fifth-order system for $\left(\hat{\lambda}_{0}, \hat{\phi}_{1}, \hat{\kappa}_{1}, \hat{N}_{1}, \hat{Q}_{1}\right)$, which we address first. $\hat{\kappa}_{1}, \hat{r}_{1}$ and $\hat{Q}_{1}$ can be eliminated from this system through substitution, to yield the equations

$$
\begin{aligned}
\hat{\phi}_{1 \hat{s} \hat{s} \hat{s}} & =\hat{N}_{1} \hat{\phi}_{1 \hat{s}}+2 \hat{\lambda}_{0} \sin \hat{\phi}_{1} / \sin \tilde{s}_{c} \\
\hat{N}_{1 \hat{s}} & =2 \hat{\lambda}_{0} \cos \hat{\phi}_{1} / \sin \tilde{s}_{c}-\hat{\phi}_{1 \hat{s}} \hat{\phi}_{1 \hat{s} \hat{s}} \\
\hat{\lambda}_{0 \hat{s}} & =\left(\cos \hat{\phi}_{1}-\cos \tilde{s}_{c}\right) / \sin \tilde{s}_{c}
\end{aligned}
$$

together with the six boundary conditions, obtained from matching to (3.15),

$$
\hat{\phi}_{1} \rightarrow \pm \tilde{s}_{c}, \quad \text { as } \quad \hat{s} \rightarrow \pm \infty, \quad \hat{N}_{1} \rightarrow 0 \quad \text { and } \quad \hat{\lambda}_{0} \rightarrow 0 \quad \text { as } \quad \hat{s} \rightarrow \pm \infty
$$

We note that the non-linear equations $(3.22 a-c)$ are symmetric under $\hat{s} \rightarrow-\hat{s}, \hat{\phi}_{1} \rightarrow-\hat{\phi}_{1}$, $\hat{\lambda}_{0} \rightarrow-\hat{\lambda}_{0}$ and $\hat{N}_{1} \rightarrow \hat{N}_{1}$ and that the conditions at the contact point $\hat{\phi}_{1}(0)=\hat{\phi}_{1 \hat{s}}(0)=0$ are automatically satisfied.

The inner region allows the slope $\phi$ to change sign smoothly across the contact point, thereby regularising the jump in this quantity in the outer solution (such as in $\bar{z}_{\tilde{s}}$ and $\bar{\kappa}$ ) between regions $\mathscr{D}_{\text {out }}^{-}$and $\mathscr{D}_{\text {out }}^{+}$.

The system (3.22) can be linearised about the far-field limits. Let $\hat{\phi}_{1}= \pm \tilde{s}_{c}+\tilde{\phi}$. We then have $\tilde{\phi}_{\text {ssss }} \sim-2 \tilde{\phi}$ as $\hat{s} \rightarrow \pm \infty$, where $\tilde{\phi}$ and its derivatives tend to zero as $\hat{s} \rightarrow \pm \infty$, leading to

$$
\tilde{\phi}=A_{1}^{ \pm} \exp \left[\mp\left(\hat{s}-A_{2}^{ \pm}\right) \frac{1 \pm i}{2^{1 / 4}}\right]+c . c . \quad \text { as } \quad \hat{s} \rightarrow \pm \infty,
$$

with $A_{1}^{ \pm}$and $A_{2}^{ \pm}$undetermined constants; c.c. denotes complex conjugate.

Suppressing the two growing far-field eigenmodes yields solutions with two degrees of freedom (one of which is equivalent to translational invariance) of the form

$$
\tilde{\phi}=\exp \left[\mp 2^{-1 / 4} \hat{s}\right]\left(\alpha \cos \left(\frac{\hat{s}}{2^{1 / 4}}\right)+\beta \sin \left(\frac{\hat{s}}{2^{1 / 4}}\right)\right) \quad \text { as } \hat{s} \rightarrow+\infty,
$$

for constants $\alpha$ and $\beta$, with the appropriately symmetric solution as $\hat{s} \rightarrow-\infty$. Such decaying oscillatory overshoot is evident in Figure 5(a). 
Rather than proceed to solve (3.22) numerically, we now look at the simpler special case for which $\tilde{s}_{c}$ is small, so that the trigonometric functions in (3.22) can be approximated to leading order by polynomials.

\subsection{Inner region, case II (i.e. $C^{-1 / 4} \ll \tilde{s}_{c} \ll 1$ )}

Here we consider a buckled vesicle in the case of small deformations (which does not exclude large variations of slopes and stresses within the inner region). Motivated by numerical simulations (see Figure 5), and considering the limiting form of (3.21)-(3.22) for $\tilde{s}_{c} \ll 1$, we write

$$
\begin{array}{lrl}
\tilde{s}=\tilde{s}_{c}+C^{-1 / 4} \hat{s}, & F=F_{0} C^{1 / 4}+F_{1}+\cdots, \\
r=\sin \tilde{s}_{c}+C^{-1 / 4}\left(\hat{s}+\tilde{s}_{c}^{2} r_{1}\right)+C^{-1 / 2} \tilde{s}_{c} r_{2}+\cdots, & \kappa=C^{1 / 4} \tilde{s}_{c} \kappa_{1}+\kappa_{2}+\cdots, \\
z=C^{-1 / 4} \tilde{s}_{c} z_{1}+C^{-1 / 2} z_{2}+\cdots, & Q=C^{1 / 2} \tilde{s}_{c} Q_{1}+C^{1 / 4} Q_{2}+\cdots, \\
\phi=\tilde{s}_{c} \phi_{1}+C^{-1 / 4} \phi_{2}+\cdots, & T=-1+\tilde{s}_{c} C^{-1 / 4} T_{0}+C^{-1 / 2} T_{1} \\
& \\
p=p_{0} \delta(\hat{s}) C^{1 / 2}+\cdots, & +C^{-3 / 4} T_{2} / \tilde{s}_{c}+\cdots, \\
\lambda= & +\tilde{s}_{c} C^{-1 / 4} \lambda_{0}+C^{-1 / 2} \lambda_{1} \\
& +C^{-3 / 4} \lambda_{2} / \tilde{s}_{c}+\cdots,
\end{array}
$$

with $N_{1}=T_{1}-\lambda_{1}$ and $N_{2}=T_{2}-\lambda_{2}$.

After substitution of (3.26) in (3.18) and Taylor-expansion with respect to the small parameters $C^{-1 / 4}$ and $\tilde{s}_{c}$, different terms appear with coefficients of the form $C^{n / 4} \tilde{S}_{c}^{m}$ (where $(n, m) \in \mathbb{Z}^{2}$ ). Ordering the terms for $C^{-1 / 4} \ll \tilde{s}_{c}$, the two largest contributions are unambiguously $\mathcal{O}\left(C \tilde{s}_{c}^{2}\right)$ and $\mathcal{O}\left(C^{3 / 4} \tilde{s}_{c}\right)$ respectively, whereas the third largest contribution cannot be determined without making some further assumption on the size of $\tilde{s}_{c}$.

Only the second term in the right-hand side of $(3.18 e)$ is $\mathcal{O}\left(C \tilde{s}_{c}^{2}\right)$, hence $\hat{T}_{0}=\hat{\lambda}_{0}$ straightforwardly. Proceeding to $\mathcal{O}\left(C^{3 / 4} \tilde{S}_{c}\right)$ in $(3.18)$, we have

$$
\begin{aligned}
r_{1 \hat{s}} & =-\frac{1}{2} \phi_{1}^{2}, & & \kappa_{1 s}=-Q_{1}, \\
z_{1 \hat{s}} & =\phi_{1}, & & Q_{1 \hat{s}}=-N_{1} \kappa_{1}-2 \lambda_{0} \phi_{1}, \\
\phi_{1 \hat{s}} & =\kappa_{1}, & & N_{1 \hat{s}}=2 \lambda_{0}, \\
\lambda_{0} & =r_{1}+\frac{1}{2} \hat{s} . & &
\end{aligned}
$$

Once again, the equations for $r_{1}$ and $z_{1}$ decouple from the fifth-order system for $\left(\lambda_{0}, \phi_{1}, \kappa_{1}, N_{1}, Q_{1}\right)$, which we solve first. The equation for $Q_{1}$ can be integrated, with the conditions $Q_{1} \rightarrow 0$ and $N_{1} \rightarrow 0$ as $\hat{s} \rightarrow \infty$ yielding $Q_{1}=-N_{1} \phi_{1}$. Eliminating $\kappa_{1}, r_{1}$ 
and $Q_{1},(3.27)$ is reduced to the fourth-order system

$$
\begin{aligned}
\phi_{1 \hat{s} \hat{s}} & =N_{1} \phi_{1}, \\
N_{1 \hat{s}} & =2 \lambda_{0}, \\
2 \lambda_{0 \hat{s}} & =1-\phi_{1}^{2},
\end{aligned}
$$

which can also be written in the compact form

$$
\left(\frac{\phi_{1 \hat{s} \hat{s}}}{\phi_{1}}\right)_{\hat{s} \hat{s}}=1-\phi_{1}^{2} .
$$

The four boundary conditions

$$
\phi_{1}(0)=N_{1 \hat{s}}(0)=0, \quad \text { and } \quad \phi_{1} \rightarrow \pm 1 \quad \text { as } \quad \hat{s} \rightarrow \pm \infty
$$

consistent with (3.23), enforce the symmetry conditions about $\hat{s}=0$ (as pointed out above, $\phi_{1}$ and $N_{1}$ are respectively odd and even functions of $\hat{s}$ ) and the matching conditions with the outer regions. We note that an equivalent inner-region ODE emerges from Reissner's formulation for a buckled shell (Parker \& Wan, 1984).

The symmetry conditions imply that $Q$ is odd and hence that $Q_{1}\left(0^{+}\right)=Q_{1}\left(0^{-}\right)=0$. Surprisingly, the jump in $Q$ (which is proportional to the vertical force exerted by the plates on the vesicle, according to $(2.13 b)$ ) therefore appears only at higher order in $C$ in this case.

We obtain a numerical solution of (3.29)-(3.30) by shooting backwards from the linearised solution (3.25) of the system (3.22) in the far field and adjusting the two parameters $\alpha$ and $\beta$ so that the solution satisfies $\phi_{1}(0)=N_{1 s}(0)=0$.

Figure 5 compares the numerical solution obtained by solving the full system (2.11), for large values of $C$, to the leading-order asymptotic solution of (3.29-3.30). Figure 5(a) shows how the slope of the vesicle goes from negative values to positive ones as it crosses $\tilde{s}_{c}$, thus regularising the discontinuous solution $(3.6 b)$ in the outer region and enforcing the boundary condition $\phi=0$ at the contact point. The variables $\phi$ and $\kappa / C^{1 / 4}$ (Figure 5(b)) converge quickly towards the leading-order asymptotic solutions $\tilde{s}_{c} \phi_{1}$ and $\tilde{s}_{c} \kappa_{1}$, respectively, in the neighbourhood of $\tilde{s}_{c}$ (for $\hat{s} \lesssim 2$ ). For $|\hat{s}| \gtrsim 2$ the contribution of the leading-order term $\kappa_{1}$ becomes smaller than higher order terms and the convergence is much slower, both for $\kappa$ and $\phi$. The convergence towards the leading-order asymptotics is slower for the meridional stress component $N_{\phi}$ (Figure 5(c)) than for the azimuthal stress component $N_{\theta}$ (Figure 5(d)), arguably because the former (of order $C^{-1 / 2}$ ) is more sensitive to the $\mathcal{O}\left(C^{-3 / 4}\right)$ forcing of the outer solution in $\mathscr{D}_{\text {out }}^{+}(3.15)$ than the latter (of order $\tilde{s}_{c} C^{-1 / 4}$ ). To leading order, the out-of-plane stress resultant $Q$ is an odd function of $\hat{s}$, with negative (resp. positive) values in the neighbourhood $\hat{s}<0$ (resp. $\hat{s}>0$ ) of the contact point, as shown in Figure 5(e). The $\tilde{s}_{c} Q_{1}$, which denotes the $\mathcal{O}\left(C^{-1 / 2}\right)$ term in the expansion for $Q$ in the inner region, sums to zero when integrated over the whole inner region, consistent with a jump in $\bar{Q}$ of $\mathcal{O}\left(C^{-3 / 4}\right)$ between both outer regions (see $(3.2 b)$ and (3.8)). To leading order, $Q$ acts like an effective bending dipole at the contact point contributing zero net force on the vesicle. 
Integrating the equation for $z_{1}$ in (3.27) yields the shape of the vesicle to leading order in the inner region. Subtracting the expression for the leading-order outer solution $\bar{z}_{0}$ (3.6a), with $\left|\tilde{s}-\tilde{s}_{c}\right|=\mathcal{O}\left(C^{-1 / 4}\right)$ (i.e. (3.16)), from the limits of $z_{1}$ as $\hat{s} \rightarrow \pm \infty$ yields the first-order correction to $z$ in the outer region, as defined in $(3.2 c)$,

$$
\bar{z}_{1}^{ \pm}= \pm \tilde{s}_{c} \int_{0}^{\infty}\left(\phi_{1}(\hat{s})-1\right) \mathrm{d} \hat{s} \approx \mp 0.1769 \tilde{s}_{c} .
$$

Using $(2.13 c)$, the second term in the expansion of $h(3.1)$ is then

$$
h_{1}=2 \bar{z}_{1}^{+} \approx-0.3538 \tilde{s}_{c}
$$

We construct a composite solution

$$
\tilde{z}_{\text {comp }}(\widetilde{s})=\bar{z}_{0}^{ \pm}(\widetilde{s})+C^{-1 / 4}\left(\bar{z}_{1}^{ \pm}+\tilde{s}_{c} z_{1}\right)-\tilde{s}_{c}\left(\tilde{s}-\tilde{s}_{c}\right), \quad \tilde{r}_{\text {comp }}(\widetilde{s})=\sin \tilde{s},
$$

valid in the whole domain. It agrees very well with the numerical solution obtained by solving the full system (2.11)-(2.13) for $C=10^{7}$ (Figure 3), indicating that the shape of the buckled vesicle can be described well by the leading-order asymptotics. However, the force acting on the vesicle is still unknown at this stage, and can be determined only by looking at higher-order contributions. A similar difficulty was reported by Kriegsmann \& Lange (1980), Graff et al. (1985) and Evkin \& Kalamkarov (2001), and we expect it also in case I.

\subsubsection{A solvability condition for the force $F_{0}$ on the vesicle, case IIb}

As described above, much insight can be obtained by considering $\mathcal{O}\left(C \tilde{s}_{c}^{2}\right)$ and $\mathcal{O}\left(C^{3 / 4} \tilde{s}_{c}\right)$ contributions in (2.11), but higher-order terms prove to be necessary to derive the forcedisplacement relationship $F$ versus $h$. The next contributions in $(2.11)$ are $\mathcal{O}\left(C^{3 / 4} \tilde{S}_{c}^{3}\right)$ and $\mathcal{O}\left(C^{1 / 2}\right)$ (there are no terms of $\mathcal{O}\left(C^{3 / 4} \tilde{s}_{c}^{2}\right)$ ). Restricting our analysis to the case $C^{-1 / 4} \ll \tilde{s}_{c} \ll C^{-1 / 12}$ (henceforth referred to as case IIb) allows us to neglect the $\mathcal{O}\left(C^{3 / 4} \tilde{s}_{c}^{3}\right)$ contribution (essentially, non-linearities arising from trigonometric terms in (2.11)) while accounting for the rest of the physics. In this case, to $\mathcal{O}\left(C^{1 / 2}\right)$ in $(2.11)$, we have

$$
\begin{aligned}
r_{2 \hat{s}} & =-\phi_{1} \phi_{2}-\lambda_{0} \\
z_{2 \hat{s}} & =\phi_{2} \\
\phi_{2 \hat{s}} & =\kappa_{2} \\
\kappa_{2 \hat{s}} & =-Q_{2}-\kappa_{1} \\
Q_{2 \hat{s}} & =p_{0} \delta(\hat{s})-N_{1} \kappa_{2}-N_{2} \kappa_{1}-\left(N_{1}+2 \lambda_{1}\right) \phi_{1}+2 \lambda_{0} \hat{s} \phi_{1}-Q_{1}-2 \lambda_{0} \phi_{2} \\
N_{2 \hat{s}} & =2 r_{2}-4 \hat{s} r_{1}-\hat{s}^{2}
\end{aligned}
$$

The equation for $Q_{2}$ can be integrated to give

$$
Q_{2}=p_{0} H(\hat{s})-\left(N_{1} \phi_{2}+N_{2} \phi_{1}\right)+\text { Constant },
$$


where $H(\hat{s})$ is the Heaviside function. As $\hat{s} \rightarrow-\infty, Q_{2} \rightarrow 0, N_{1} \rightarrow 0$ and $N_{2} \rightarrow 0$ (see below), so the constant of integration is zero. Furthermore, as $\hat{s} \rightarrow 0^{-}, \phi_{1} \rightarrow 0$ and $\phi_{2} \rightarrow 0$ (by definition, all terms in the expansion for $\phi$ vanish at the contact point $\hat{s}=0$ ), so that $Q_{2}\left(0^{-}\right)=0$. Thus the first two terms in the expansion for $Q(3.26 c)$ are zero as $\hat{s} \rightarrow 0^{-}$.

The matching conditions to $\mathscr{D}_{\text {out }}^{-}$, as derived in (3.15), lead to $\phi_{2} \rightarrow 0$ and $N_{2} \rightarrow 0$ as $\hat{s} \rightarrow-\infty$.

The matching conditions to $\mathscr{D}_{\text {out }}^{+}$are $\phi_{2} \rightarrow 0, N_{2} \rightarrow-B^{+} / \tilde{s}_{c}$ (since $\lambda_{2} / \tilde{s}_{c} \sim \bar{\lambda}_{1}, T_{2} \rightarrow 0$, $N_{2}=T_{2}-\lambda_{2}$ and $\bar{\lambda}_{1}(\tilde{s}) \sim B^{+} / \tilde{s}_{c}^{2}$ as $\tilde{s} \rightarrow \tilde{s}_{c}$ ) and $Q_{2} \rightarrow 0$ (since $\bar{Q}_{0}=0$ ) for $\hat{s} \rightarrow \infty$. Note that the forcing on the inner region comes only from $\mathscr{D}_{\text {out }}^{+}$, at this order.

Taking the limit $\hat{s} \rightarrow \infty$ in (3.35) using (3.30) allows us to determine $B^{+}$:

$$
B^{+}=-\tilde{S}_{c} p_{0}
$$

The second-order problem (3.34) thus reduces to a fourth-order system of the form

$$
\mathscr{L} X=W
$$

where

$$
\mathscr{L}=\left(\begin{array}{cccc}
\partial_{\hat{s}} & -1 & 0 & 0 \\
0 & \partial_{\hat{s}} & 2 \phi_{1} & 0 \\
0 & 0 & \partial_{\hat{s}} & -1 \\
-\phi_{1} & 0 & -N_{1} & \partial_{\hat{s}}
\end{array}\right), \quad X=\left(\begin{array}{c}
N_{2} \\
N_{2 \hat{s}} \\
\phi_{2} \\
\phi_{2 \hat{s}}
\end{array}\right) \quad \text { and } \quad W=\left(\begin{array}{c}
0 \\
2 \hat{s} \phi_{1}^{2}-6 r_{1}-3 \hat{s} \\
0 \\
-p_{0} H(\hat{s})-\phi_{1 \hat{s}}
\end{array}\right)
$$

and $X$ satisfies the boundary conditions ( $X_{i}$ denotes the $i^{\text {th }}$ component of $X$ )

$$
\begin{aligned}
& X_{1} \rightarrow 0 \quad \text { and } \quad X_{3} \rightarrow 0 \text { for } \hat{s} \rightarrow-\infty \\
& X_{1} \rightarrow p_{0} \text { and } \quad X_{3} \rightarrow 0 \text { for } \hat{s} \rightarrow+\infty
\end{aligned}
$$

in order to ensure the matching of the solution to $\mathscr{D}_{\text {out }}^{-}$and $\mathscr{D}_{\text {out }}^{+}$(for $\left.\tilde{s}_{c} \ll 1\right)$.

Rather than solve (3.37) directly, we formulate an adjoint problem and a solvability condition that allow us to derive the forcing term $p_{0}$ and the jump in $Q$. We define the linear operator

$$
\mathscr{L}^{\dagger}=\left(\begin{array}{cccc}
-\partial_{\hat{s}} & 0 & 0 & -\phi_{1} \\
-1 & -\partial_{\hat{s}} & 0 & 0 \\
0 & 2 \phi_{1} & -\partial_{\hat{s}} & -N_{1} \\
0 & 0 & -1 & -\partial_{\hat{s}}
\end{array}\right)
$$

with the property that, for any column vector of functions $Y$,

$$
\sum_{i=1}^{4} Y_{i}(\mathscr{L} X)_{i}-X_{i}\left(\mathscr{L}^{\dagger} Y\right)_{i}=\frac{\mathrm{d}}{\mathrm{d} s}\left(\sum_{i=1}^{4} X_{i} Y_{i}\right)
$$

In addition, we define the column vector $Y^{*}=\left(-N_{1 \hat{s} \hat{s}},-N_{1 \hat{s}}, 2 \phi_{1 \hat{s} \hat{s}}, 2 \phi_{1 \hat{s}}\right)^{T}$ constructed 
from the solution of the first-order problem (3.28), the components of which satisfy

$$
\begin{array}{lll}
Y_{2} \rightarrow 0 & \text { and } \quad Y_{4} \rightarrow 0 \\
Y_{1} \rightarrow 0 & \text { for } \quad \hat{s} \rightarrow+\infty
\end{array}
$$

It is then straightforward to verify that $\mathscr{L}^{\dagger} Y^{*}=0$. Taking the inner product between $Y^{*}$ and $\mathscr{L} X$ in (3.37), integrating by parts following (3.41) and noting that the boundary conditions vanish due to (3.39) and (3.42), we obtain a solvability condition of the form $\sum_{i=1}^{4} \int_{-\infty}^{\infty} W_{i} Y_{i}^{*} \mathrm{~d} \hat{s}=0$, i.e.

$$
\int_{-\infty}^{\infty}\left[-N_{1 \hat{s}}\left(2 \hat{s} \phi_{1}^{2}-6 r_{1}-3 \hat{s}\right)+2 \phi_{1 \hat{s}}\left(-p_{0} H(\hat{s})-\phi_{1 \hat{s}}\right)\right] \mathrm{d} \hat{s}=0 .
$$

All the functions at first order being known, (3.43) uniquely determines the scalar $p_{0}$. Now (2.12) leads to $F_{0}=-2 \pi \tilde{s}_{c} p_{0}$ (using $\tilde{s}_{c} \ll 1$ and the identity $\delta\left(\tilde{s}-\tilde{s}_{c}\right)=C^{1 / 4} \delta(\hat{s})$ ), so that using (3.7) to eliminate $\tilde{s}_{c}$ and evaluating (3.43) numerically, we have

$$
p_{0} \approx-3.966 \text { and } F_{0}=-2 \pi p_{0} \sqrt{2-h_{0}} .
$$

Figure 6 shows that the leading-order force-displacement relationship (3.44b) agrees well with the full numerical solution of buckled solutions of Preston et al. (2008) as $C \rightarrow \infty$ within region II. In the context of our analysis (i.e. case IIb), (3.44) holds when the displacement of the plates from their initial position (i.e. $h=2$ ) satisfies $C^{-1 / 2} \ll 2-h \ll C^{-1 / 6}$. For $C=10^{3}$ (resp. $C=10^{6}$ ), this range corresponds to changes of the vesicle height within $1.5 \%-15 \%$ (resp. $0.05 \%-5 \%$ ) of its undeformed diameter.

Following (2.13b) and (3.44), the leading-order jump in $Q$ at the contact point, with value $C^{1 / 4} p_{0}$, is independent of $\tilde{s}_{c}$ within the whole range of validity of case IIb.

Having examined the post-buckled behaviour of the vesicle under compression, we now proceed to the case of smaller deformations where both buckled and unbuckled solutions exist.

\subsection{Inner region, case III (i.e. $C^{-1 / 4} \sim \tilde{s}_{\mathcal{C}} \ll 1$ )}

When $\tilde{s}_{c}$ becomes small, the inner region around the buckling ring encompasses the pole (Figure 7(c)) and deformations are weaker than those captured in the expansion (3.26). Since $\phi=\mathcal{O}\left(\tilde{s}_{c}\right)$ in the inner region, we choose, for $\tilde{s}_{c} \equiv C^{-1 / 4} \breve{s}_{c}, \check{S}_{c}=\mathcal{O}(1)$,

$$
\begin{array}{rlrl}
\tilde{s} & =C^{-1 / 4} \check{s}, \\
r & =C^{-1 / 4} \hat{r}_{1}+C^{-1 / 2} \hat{r}_{2}+\cdots, & & \kappa=\hat{\kappa}_{1}+C^{-1 / 4} \hat{\kappa}_{2}+\cdots \\
z & =C^{-1 / 2} \hat{z}_{1}+C^{-3 / 4} \hat{z}_{2}+\cdots, & & Q=C^{1 / 4} \hat{Q}_{1}+\hat{Q}_{2}+\cdots \\
\phi & =C^{-1 / 4} \hat{\phi}_{1}+C^{-1 / 2} \hat{\phi}_{2}+\cdots, & T=-1+\hat{T}_{0} C^{-1 / 4}+\hat{T}_{1} C^{-1 / 2}+\hat{T}_{2} C^{-3 / 4}+\cdots \\
p & =\check{p}_{0} \delta\left(\check{s}-\check{s}_{c}\right) C^{1 / 2}+\cdots, & \lambda=1+\hat{\lambda}_{0} C^{-1 / 4}+\hat{\lambda}_{1} C^{-1 / 2}+\hat{\lambda}_{2} C^{-3 / 4}+\cdots,
\end{array}
$$


where hats denote functions of $\breve{s}$. In contrast to (3.19)-(3.26), the amplitude of the leadingorder pressure term $\check{p}_{0}$ is found to depend on $\check{s}_{c}$ (see below), hence the different notation. With the scaling (3.45a), note that the re-scaled inner equations are identical to (3.18), with the exception of $(3.18 \mathrm{~g})$.

As shown in (3.26), for $C^{-1 / 4} \ll \tilde{s}_{c} \ll 1$, the scaling for the out-of-plane stress resultant is $Q(\tilde{s}) \propto C^{1 / 2} \tilde{S}_{c}$ and for the pressure (forcing term) $p(\tilde{s}) \propto C^{1 / 4} \delta\left(\tilde{s}-\tilde{S}_{c}\right)$, so that $p$ does not contribute to leading order. However, when $\tilde{s}_{c}=\mathcal{O}\left(C^{-1 / 4}\right)$ then $Q(\tilde{s})$ and $p(\tilde{s})$ are of the same order. We therefore expect the jump in $Q$ to appear at leading order, unlike in cases I and II where the leading-order inner solution is continuous. The force (2.12) is now $\mathcal{O}(1)$, so we expect $F_{0}=0$ in $(3.26 a)$.

\subsubsection{Leading order}

Substituting the expansion (3.45) into $(2.11 g)$ yields

$$
\hat{r}_{1}=\check{s}, \quad \hat{r}_{2}=\check{s} \hat{\lambda}_{0} \quad \text { and } \quad \hat{r}_{3}=\check{s} \hat{\lambda}_{1}-\check{s}^{3} / 6
$$

Eliminating $r$ between $(2.11 a)$ and $(2.11 g)$ leads to $2 \hat{\lambda}_{0}+\check{s}_{\lambda}{ }_{\check{s}}=0$, for which the only non-diverging solution as $\check{S} \rightarrow 0$ is $\hat{\lambda}_{0}=0$. To leading order, $(2.11 f)$ reduces to $\hat{T}_{0 s}=0$ and therefore $\hat{T}_{0}=0$ (in order to satisfy the matching conditions with the outer region). The leading-order equations are thus

$$
\begin{aligned}
\hat{z}_{1 \check{s}} & =\hat{\phi}_{1}, \\
\hat{\phi}_{1 \check{s}} & =\hat{\kappa}_{1}, \\
\frac{1}{\check{s}}\left(\check{s} \hat{\kappa}_{1}\right)_{\check{s}} & =+\frac{\hat{\phi}_{1}}{\check{s}^{2}}-\hat{Q}_{1}, \\
\frac{1}{\check{s}}\left(\check{s} \hat{Q}_{1}\right)_{\check{s}} & =\check{p}_{0} \delta\left(\check{s}-\check{s}_{c}\right)-\left(\hat{T}_{1}-\hat{\lambda}_{1}\right) \hat{\kappa}_{1}-\frac{\hat{T}_{1}+\hat{\lambda}_{1}}{\check{s}} \hat{\phi}_{1}, \\
\hat{T}_{1 \check{s}} & =-\frac{\hat{\phi}_{1}^{2}}{2 \check{s}}+\frac{\check{s}}{2}, \\
\hat{\lambda}_{1 \check{s}} & =-\frac{\hat{\phi}_{1}^{2}}{2 \check{s}}+\frac{\check{s}}{2}-\frac{2 \hat{\lambda}_{1}}{\check{s}} .
\end{aligned}
$$

Boundary conditions are obtained by (a) expanding (3.47) near the origin for small $\breve{s}$ (using the axisymmetry assumption), imposing that the slope and height vanish at the contact point and by (b) matching the slope and the curvature with the outer solution for $\check{s} \rightarrow \infty$ :

$$
\begin{aligned}
& \hat{\phi}_{1} \sim K_{0} \check{s}, \quad \hat{\kappa}_{1} \sim K_{0}, \quad \hat{\lambda}_{1}=\mathcal{O}\left(\check{s}^{2}\right), \quad \hat{Q}_{1} \sim \mathcal{O}(\check{s}) \quad \text { as } \check{s} \rightarrow 0^{+}, \\
& \hat{\phi}_{1}=\hat{z}_{1}=0, \quad \text { for } \check{s}=\check{s}_{c}, \\
& \hat{\phi}_{1} \sim \check{s} \quad \text { and } \quad \hat{\kappa}_{1} \sim 1 \quad \text { as } \check{s} \rightarrow \infty,
\end{aligned}
$$

where $K_{0}$ is some undetermined constant. By imposing (3.48a) for small $\check{s}$, we suppress two growing modes that diverge like $1 / \breve{s}^{2}$. The boundary conditions (3.48) are valid whether or not the vesicle buckles (with $K_{0}=0$ for an unbuckled vesicle). 
As in (3.34), the equation for $\hat{Q}_{1}$ can be integrated to give

$$
\hat{Q}_{1}=\check{p}_{0} H\left(\check{s}^{-} \check{s}_{c}\right) \check{S}_{c} / \check{s}-\left(\hat{T}_{1}-\hat{\lambda}_{1}\right) \hat{\phi}_{1}+\text { Constant. }
$$

Since $\hat{Q}_{1}\left(0^{+}\right)=\hat{\phi}_{1}\left(0^{+}\right)=0$, the constant of integration is zero. The equation for $\hat{z}_{1}$ decouples, so that the system is actually fourth order and reduces to

$$
\begin{aligned}
& \hat{\phi}_{1 \check{s}}=\hat{\kappa}_{1}, \\
& \hat{\kappa}_{1 \check{s}}=\left(\hat{T}_{1}-\hat{\lambda}_{1}\right) \hat{\phi}_{1}-\frac{\hat{\kappa}_{1}}{\check{s}}+\frac{\hat{\phi}_{1}}{\check{s}^{2}}-\frac{\check{p}_{0} \check{s}_{c} H\left(\check{s}-\check{s}_{c}\right)}{\check{s}}, \\
& \hat{T}_{1 \check{s}}=-\frac{\hat{\phi}_{1}^{2}}{2 \check{s}}+\frac{\check{s}}{2}, \\
& \hat{\lambda}_{1 \check{s}}=-\frac{\hat{\phi}_{1}^{2}}{2 \check{s}}+\frac{\check{s}}{2}-\frac{2 \hat{\lambda}_{1}}{\check{s}},
\end{aligned}
$$

with the boundary conditions:

$$
\begin{aligned}
& \hat{\phi}_{1} \sim K_{0} \check{s}, \quad \hat{\kappa}_{1} \sim K_{0}, \quad \hat{\lambda}_{1}=\mathcal{O}\left(\check{s}^{2}\right), \\
& \hat{\phi}_{1}=0, \quad \text { for } \quad \check{s}=\check{s}_{c}, \\
& \hat{\phi}_{1} \sim \check{s} \quad \text { and } \quad \hat{\kappa}_{1} \sim 1 \quad \text { as } \quad \check{s} \rightarrow \infty .
\end{aligned}
$$

Applying the matching conditions $\hat{\phi}_{1} \sim \check{S}, \hat{T}_{1} \rightarrow 0, \hat{Q}_{1} \rightarrow 0$ and $\hat{\lambda}_{1} \sim-B^{+} C^{1 / 4} / \check{s}^{2}$ as $\check{S} \rightarrow$ $\infty$, (which are derived from (3.15) and (3.45)) to (3.49), yields $B^{+}=-\check{S}_{c} C^{-1 / 4} \check{p}_{0}=-\widetilde{s}_{c} \check{p}_{0}$ (consistent with (3.36) for the case IIb). $B^{+}$provides the correction to the spherical shape in the outer region $\mathscr{D}_{\text {out }}^{+}(3.13)-(3.14)$.

We have a total of three unknown parameters $\left(K_{0}, \check{s}_{c}\right.$ and $\left.\check{p}_{0}\right)$ and six boundary conditions for the fourth-order system (3.50). We thus expect this to be well posed if we prescribe the value of one parameter. In what follows, we prescribe the contact point $\check{S}_{c}$ (since it is actually imposed by the outer solution, to leading order). The most notable differences between this and (3.22) and (3.28) are that the system is now non-autonomous and that the forcing term $p$ appears in the leading-order equations (through $\check{p}_{0}$ ).

\subsubsection{Numerical solution of the leading-order problem}

We use Matlab's boundary value solver (bvp4c) to solve (3.50)-(3.51) for fixed values of $\check{s}_{c}$. The initial guess is constructed from the composite solution (3.33) for large $\check{s}_{c}$. We proceed with a continuation method on $\check{S}_{c}$ and find two solution branches, corresponding to a buckled and unbuckled vesicle respectively. The unknown parameter $\check{p}_{0}$, corresponding to the jump in $\hat{Q}_{1}$ at the contact point, is plotted against $\check{s}_{c}$ in Figure $8\left(\right.$ a). As $\check{s}_{c} \rightarrow \infty$, the buckled solution matches that of case II (result not shown) and $\check{p}_{0} \rightarrow \tilde{p}_{0} \approx 3.966$. The singularity of $\check{p}_{0}$ as $\check{s}_{c} \rightarrow 0$ is due to the contact ring shrinking to a point. The behaviour of the vesicle in this limit is discussed in Section 3.5 below. The leading-order force $F \approx F_{1}=-2 \pi \check{S}_{c} \check{p}_{0}$ (following (2.12) and (3.45)) is plotted in Figure 8(b). For all values of $\check{s}_{c}$, it is easier to compress a buckled vesicle than a non-buckled one.

Figure 9 shows a comparison of the unbuckled and buckled solutions (Figures 9(a)-(b) and Figures (c)-(d) respectively) for $\check{s}_{c}=3$. In the unbuckled case the vesicle is flat in a 

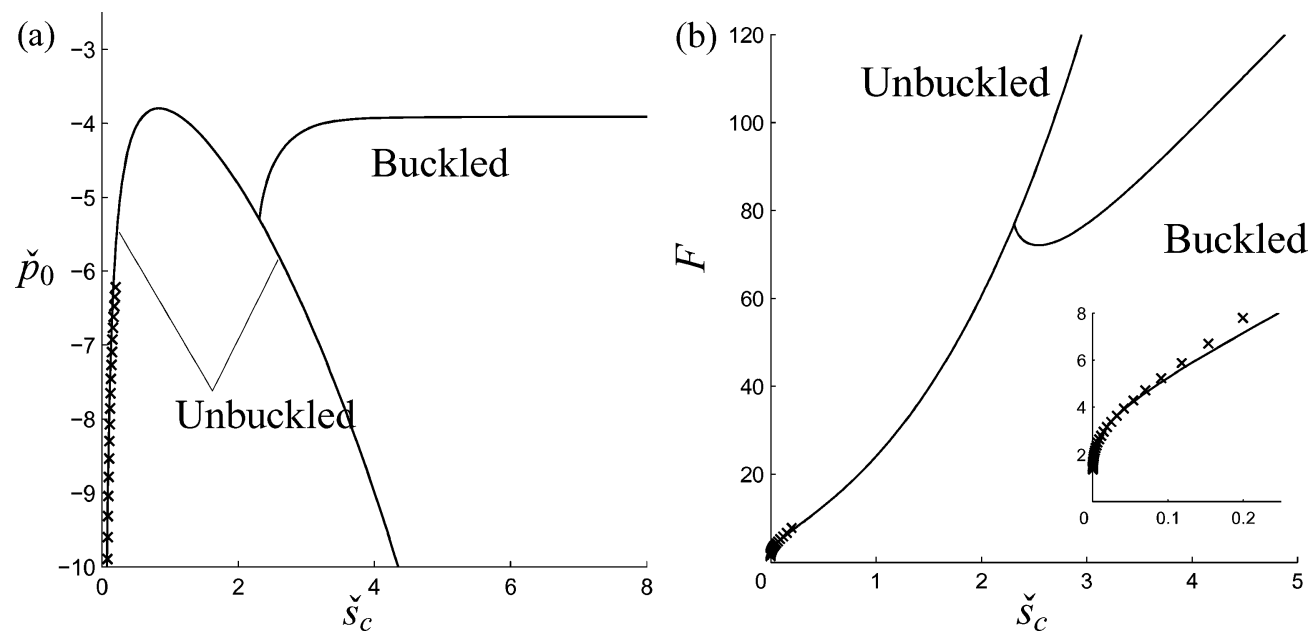

Figure 8. Case III. Amplitude of the pressure $\check{p}_{0}$ (or equivalently jump in $Q$ ) at the contact point (a) and force exerted on the vesicle (b) plotted against $\breve{s}_{c}$ to leading order in the distinguished limit $\check{S}_{c} \ll C^{1 / 4}$ for both the buckled and the unbuckled solution. The solid lines are the numerical solution of (3.50)-(3.51) and the symbols (crosses) refer to the asymptotic solution (3.69) derived for $\check{s}_{c} \ll 1$ (case IV), (2.12). The inset in (b) is a blow up of the region $\check{s}_{c} \ll 1$.

finite region $\check{s}<\check{S}_{c}$, in which the out-of-plane stress resultant $Q$ is zero $\left(\hat{\phi}_{1}=\hat{\kappa}_{1}=\hat{Q}_{1}=0\right)$, as expected from Preston et al. (2008). At $\check{S}=\check{s}_{c}, \hat{Q}_{1}$ jumps to a finite negative value (namely $\check{p}_{0}$ ), while the other variables are continuous. For all $\check{s}, \hat{\lambda}_{1}>0$, implying that $\lambda>1$ and hence that the vesicle is stretched in the azimuthal direction and compressed in the meridional direction. Physically, compression in the meridional direction is likely to induce buckling to a non-axisymmetric state.

In the buckled case (Figures 9(c)-(d)), the vesicle has a dimple for $0<\check{s}<\check{s}_{c}$ and a smaller jump in $\hat{Q}_{1}(\approx 4.1$, in absolute value $)$ than in the unbuckled case $(\approx 6.4$, in absolute value, for the same value of $\check{s}_{c}$ ) at $\check{s}=\check{s}_{c}$. The azimuthal stretch $\lambda<1$ for $\check{s} \lesssim 2.5$ and $\lambda>1$ elsewhere.

For both the buckled and unbuckled cases, $\hat{\phi}_{1}, \hat{\kappa}_{1}, \hat{\lambda}_{1}, \hat{T}_{1}$ and $\hat{Q}_{1}$ relax towards their asymptotic values (corresponding to the undeformed spherical solution) for $\check{S}^{\prime} \check{S}_{c}$. Here $\left(\check{s}_{c}=3\right)$, most of the changes occur for $\check{s}_{c}<\check{s}<2 \check{s}_{c}$.

Integrating (3.47a) between 0 and $\check{s}$ and matching the limit as $\check{s} \rightarrow \infty$ with the inner limit of the outer solution (3.17) yields

$$
\bar{z}_{1}^{ \pm}=0 \quad \text { and } \quad \bar{z}_{2}^{ \pm}=\int_{0}^{ \pm \infty}\left(\hat{\phi}_{1}-\hat{t}\right) \mathrm{d} \hat{t}-\frac{1}{2} \check{s}_{c}^{2} .
$$

The terms in the expansion of $h(3.1)$ are thus, using the boundary condition $h=2 z(\pi / 2)$ in $(2.13 c)$,

$$
h_{0}=2, \quad h_{1}=0 \quad \text { and } \quad h_{2}=2 \bar{z}_{2}^{+}=-\check{s}_{c}^{2}+2 \int_{0}^{\infty}\left(\hat{\phi}_{1}-\hat{t}\right) \mathrm{d} \hat{t} .
$$



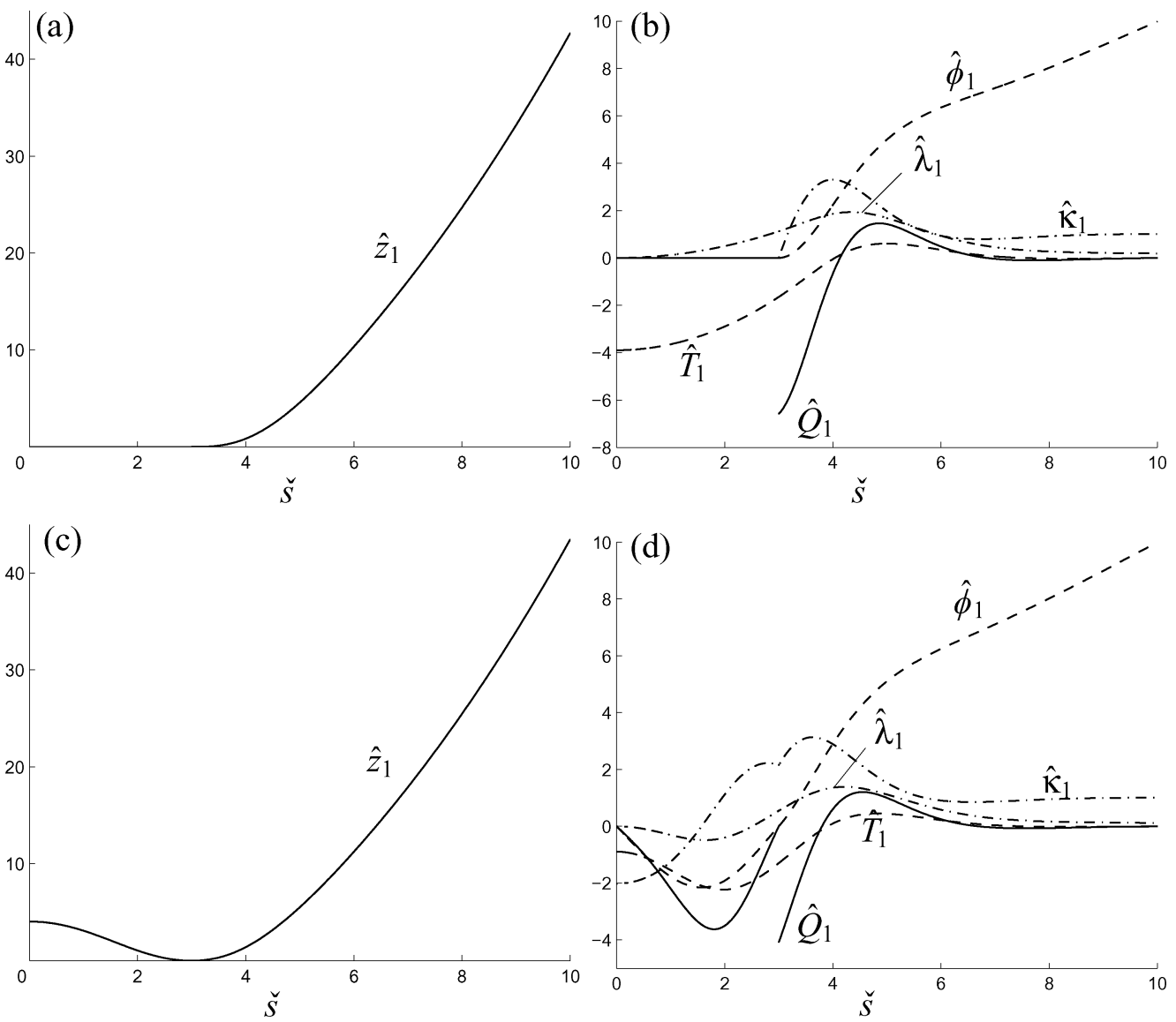

FIGURE 9. Solution of the leading-order system (3.50) and (3.51) in case III for $\check{s}_{c}=3$. (a-b) Unbuckled solution (presumably unstable); (c-d) Buckled solution.

We obtain $\mathcal{O}\left(C^{-1 / 2}\right)$ corrections for $z$ and $h$, in the case where $\tilde{s}_{c}=\mathcal{O}\left(C^{-1 / 4}\right)$, consistent with case II ((3.31)-(3.32)) where the corresponding corrective terms are in $\mathcal{O}\left(\tilde{\mathrm{s}}_{c} C^{-1 / 4}\right)$.

\subsubsection{Buckling instability}

Figure 10(a) shows the distance $m\left(\check{s}_{c}\right)=z(0)$ between the plate and the pole as the (re-scaled) location of the contact point $\check{S}_{c}$ varies. The solutions are obtained by solving (3.50, 3.51) numerically, as described above. For $\check{s}_{c}<\check{s}_{c}^{\text {crit }} \approx 2.31$, only one solution can be found: $m\left(\check{s}_{c}\right)=0$, which corresponds to an unbuckled vesicle. For $\check{s}_{c}>\breve{s}_{c}^{c r i t}$, two branches can be found: $m\left(\check{s}_{c}\right)=0$ and $m\left(\check{s}_{c}\right)>0$ (with $m$ monotonically increasing with $\check{S}_{c}$ ), corresponding to the unbuckled and buckled states respectively. To leading order as $C \gg 1$, the buckling instability is therefore supercritical when using $\breve{S}_{c}$ as the independent parameter.

However, applying (3.1) and (3.53) to determine $h$ as a function of $\check{s}_{c}$ and $C$, and using $h$ as the control parameter, the instability appears sub-critical (consistent with the 

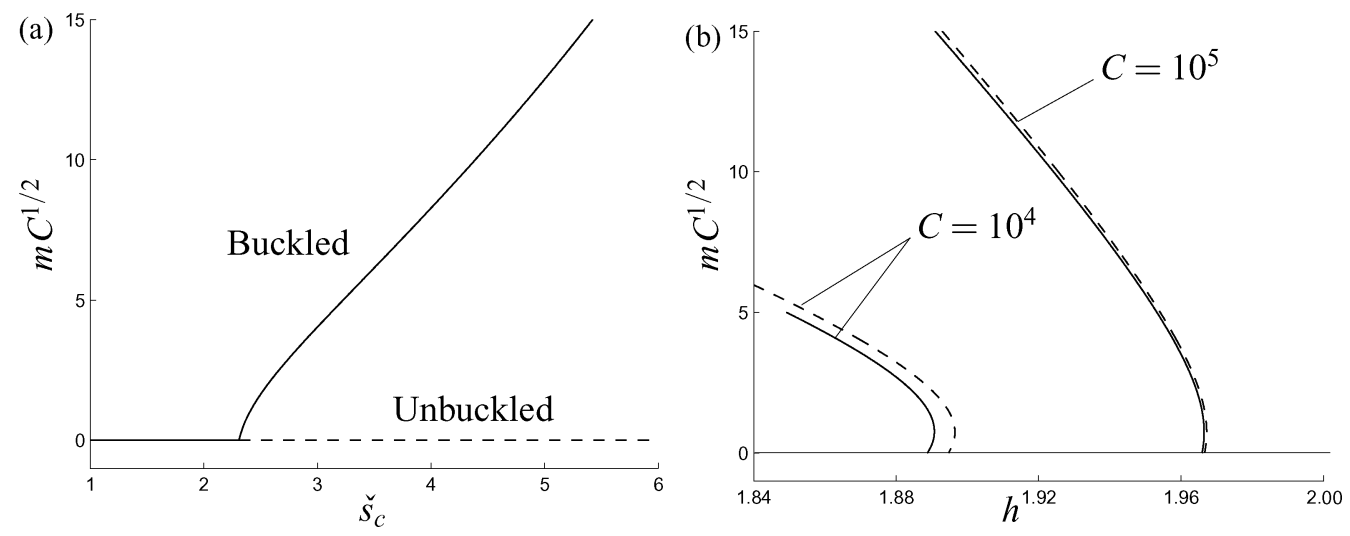

FIGURE 10. Distance $m$ between pole and plate plotted against the control parameters $\check{s}_{c}$ and $h$. (a) Leading-order asymptotic solution of (3.50 and, (3.51); (b) corresponding bifurcation diagram for $C=\left[10^{4}, 10^{5}\right]$ using the relationship between $\breve{s}_{c}$ and $h$ (3.1) and (3.53) (dashed lines). The solid lines correspond to the numerical solution of the full system (2.11)-(2.13).
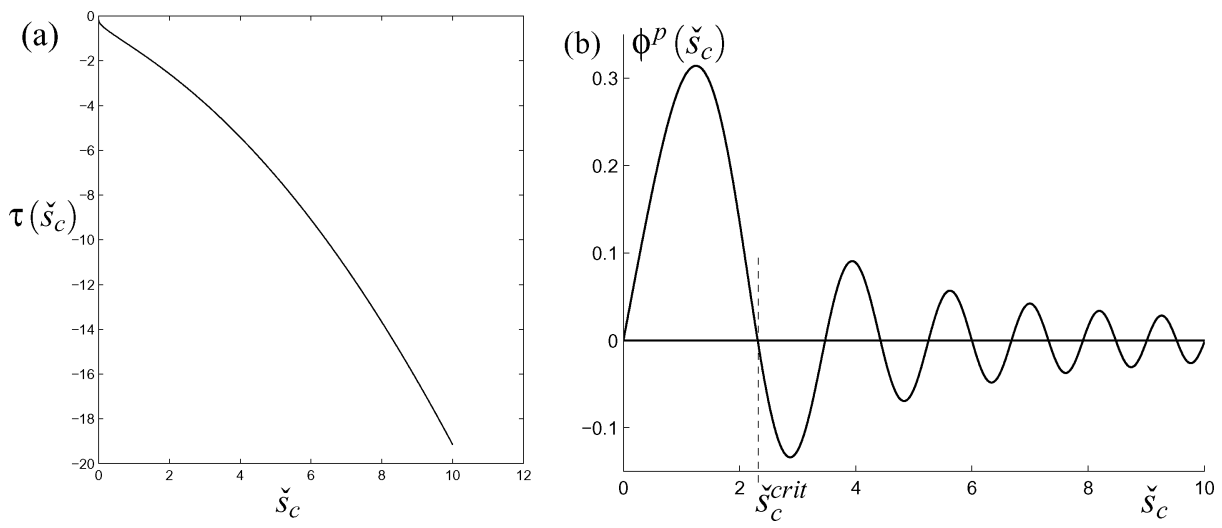

FIGURE 11. (a) Value of the isotropic stress $\hat{T}_{1}$ at the pole $\tau\left(\check{S}_{c}\right)$, obtained by solving (3.50) and (3.51) numerically; (b) Values of the small-amplitude perturbation to the unbuckled solution of (3.50) and (3.51) at the boundary $\check{s}=\check{s}_{c}$. The only possible (buckled) solutions occur when $\phi^{p}\left(\check{s}_{c}\right)=0$ (for $\left.\check{s}_{c}>0\right)$.

numerical results from Preston et al. (2008)). An example is given in Figure 10(b) for two values of $C\left(10^{4}\right.$ and $\left.10^{5}\right)$, showing the solution of the full system (2.11)-(2.13) and the leading-order asymptotics.

The value of $\breve{S}_{c}^{\text {crit }}$ can be confirmed by a linear stability analysis of the unbuckled solution of (3.50) and (3.51). For a given value of $\check{S}_{c}$, the unbuckled solution $\left(\hat{\phi}_{1}^{u b}, \hat{\kappa}_{1}^{u b}, \hat{Q}_{1}^{u b}, \hat{T}_{1}^{u b}, \hat{\lambda}_{1}^{u b}\right)$ can be computed analytically in the range $0<\check{S}<\check{S}_{c}$ :

$$
\hat{\phi}_{1}^{u b}=\hat{\kappa}_{1}^{u b}=\hat{Q}_{1}^{u b}=0, \quad \hat{T}_{1}^{u b}=\tau\left(\check{S}_{c}\right)+\check{S}^{2} / 4 \quad \text { and } \quad \hat{\lambda}_{1}^{u b}=\check{s}^{2} / 8,
$$

with $\tau\left(\check{S}_{c}\right)$ an unknown function of the parameter $\check{S}_{c} . \tau\left(\check{S}_{c}\right)$ is determined by seeking the unbuckled solution of the system (3.50) and (3.51) numerically for different values of $\check{S}_{c}$ and recording the value of $\hat{T}_{1}^{u b}$ at $\check{s}=0$ (Figure 11(a)). 
Linearising about (3.54) by writing $\hat{\phi}_{1}=\hat{\phi}_{1}^{u b}+\phi^{p}, \hat{T}_{1}=\hat{T}_{1}^{u b}+T^{p}$ and $\hat{\lambda}_{1}=\hat{\lambda}_{1}^{u b}+\lambda^{p}$ and substituting in (3.50), we obtain a linear ODE for $\phi^{p}$, valid for $0<\breve{s}<\breve{s}_{c}$,

$$
\phi_{\breve{s} \check{s}}^{p}+\frac{\phi_{\breve{s}}^{p}}{\check{s}}-\left(\frac{1}{\check{s}^{2}}+\tau\left(\check{s}_{c}\right)+\frac{\check{s}^{2}}{8}\right) \phi^{p}=0
$$

with the boundary conditions

$$
\phi^{p}(\check{s}) \rightarrow 0 \text { as } \check{s} \rightarrow 0 \quad \text { and } \phi^{p}\left(\check{s}_{c}\right)=0 .
$$

The equations for the linearised perturbations to the isotropic stress $T^{p}$ and the stretch $\lambda^{p}$ decouple and we have

$$
T_{\check{s}}^{p}=0 \quad \text { and } \quad \lambda_{\check{s}}^{p}=-2 \frac{\lambda_{\check{s}}^{p}}{\check{s}} \text {. }
$$

The general solution of (3.55) is a linear combination of Whittaker functions, one of which diverges as $\check{s} \rightarrow 0$. Since the perturbation has to remain bounded, the solution is of the form

$$
\phi^{p}(\check{s}) \equiv \frac{A}{\check{S}} W_{-\frac{1}{2} \tau\left(\check{s}_{c}\right) \sqrt{2}, \frac{1}{2}}\left(\frac{\sqrt{2}}{4} \check{s}^{2}\right),
$$

with only one non-zero constant of integration $A$. The remaining boundary condition, $\phi^{p}\left(\check{s}_{c}\right)=0$, provides a condition determining $\check{s}_{c}$. The zeros of (3.58) depend on $\tau$, which itself depends non-trivially on $\check{s}_{c}$ (Figure 11(a)). Figure 11(b) shows $\phi^{p}\left(\breve{s}_{c}\right)$ plotted against $\check{s}_{c}$. The smallest value of $\breve{s}_{c}>0$ for which $\phi^{p}\left(\check{s}_{c}\right)=0$ determines the bifurcation point $\check{s}_{c}$ crit beyond which the buckled solution exists (the following zeros corresponding to the higher modes of the buckling instability). Numerically, we find

$$
\check{S}_{c}^{c r i t} \equiv C^{1 / 4} \widetilde{S}_{c}^{c r i t} \approx 2.31,
$$

which agrees very well with the value obtained above by tracking both unbuckled and buckled branches numerically (Figure 10(a)). The prediction for the onset of the buckling instability obtained by the leading-order asymptotic analysis for $C \gg 1$ (dashed line, Figure 7(e)) is also in good agreement with the computations of Preston et al. (2008) (as reported in Figure 7(e) for $C=\left[500 ; 10^{3} ; 10^{4} ; 10^{5}\right]$ and in Figure 10(b) for $\left.C=\left[10^{4} ; 10^{5}\right]\right)$.

Having understood the behaviour of the vesicle in the regime where both bending and stretching contribute to leading order in the balance of forces, we now proceed to case IV, where stretching and bending operate on different length scales. The slow decay as $\check{s} \rightarrow \infty$ of $\hat{\lambda}_{1}$ relative to other variables (such as $\hat{Q}_{1}$ ) is already visible in Figure $9(\mathrm{~b})$ but is more pronounced when $\check{s}_{c}$ gets smaller (result not shown).

\subsection{Inner region, case IV (i.e. $C^{-1 / 2} \ll \tilde{s}_{c} \ll C^{-1 / 4} \ll 1$ )}

When $\tilde{s}_{c} C^{1 / 4} \ll 1$, we find that the inner region can be sub-divided into two regions with different length scales (Figure $7(\mathrm{~d})$ ): an $\mathcal{O}\left(\tilde{s}_{c}\right)$ region near the contact point $\left(\mathscr{R}_{(i)}\right)$ dominated by bending, and an $\mathcal{O}\left(C^{-1 / 4}\right)$ region $\left(\mathscr{R}_{(i i)}\right)$ dominated by stretching. As $C \propto\left(a / h_{s}\right)^{2}$ (see 
introduction) for linearly elastic materials, the underlying assumptions of shell theory require that $C^{-1 / 2} \ll \tilde{s}_{c}$ (thus ensuring that all the in-plane length scales of the deformation are larger than the thickness of the vesicle).

\subsubsection{Bending region $\mathscr{R}_{(i)}$, with length scale $\mathcal{O}\left(\tilde{\mathrm{S}}_{c}\right)$}

The dependent variables in region $\mathscr{R}_{(i)}$ scale as follows:

$$
\begin{aligned}
\tilde{s} & =\tilde{s}_{c} s^{*}, & & \\
r & =\tilde{s}_{c} s^{*}+\tilde{s}_{c}^{2} r_{1}^{(i)} / \ln \left(1 / \check{s}_{c}\right)+\cdots, & & \kappa=\kappa_{1}^{(i)} / \ln \left(1 / \check{s}_{c}\right)+\cdots, \\
z & =\tilde{s}_{c}^{2} z_{1}^{(i)} / \ln \left(1 / \check{s}_{c}\right)+\cdots, & & Q=Q_{1}^{(i)} /\left(\tilde{s}_{c} \ln \left(1 / \check{s}_{c}\right)\right)+\cdots, \\
\phi & =\tilde{s}_{c} \phi_{1}^{(i)} / \ln \left(1 / \check{s}_{c}\right)+\cdots, & & T=-1+T_{1}^{(i)} / \ln \left(1 / \check{s}_{c}\right)+\cdots, \\
p & =p_{0}^{(i)} \delta\left(s^{*}-1\right) /\left(\tilde{s}_{c}^{2} \ln \left(1 / \check{s}_{c}\right)\right)+\cdots, & \lambda & =1+\tilde{s}_{c}^{2} \lambda_{1}^{(i)}+\cdots .
\end{aligned}
$$

Substituting the expansion (3.60) into (2.11) yields the leading-order equations:

$$
\begin{aligned}
z_{1 s^{*}}^{(i)} & =\phi_{1}^{(i)}, \\
\phi_{1 s^{*}}^{(i)} & =\kappa_{1}^{(i)}, \\
\kappa_{1 s^{*}}^{(i)} & =-\frac{\kappa_{1}^{(i)}}{s^{*}}+\frac{\phi_{1}^{(i)}}{s^{* 2}}-Q_{1}^{(i)}, \\
Q_{1 s^{*}}^{(i)} & =p_{0}^{(i)} \delta\left(s^{*}-1\right)-\frac{Q_{1}^{(i)}}{s^{*}}, \\
T_{1 s^{*}}^{(i)} & =0, \\
\lambda_{1 s^{*}}^{(i)} & =-\frac{2 \lambda_{1}^{(i)}}{s^{*}}+\frac{s^{*}}{2},
\end{aligned}
$$

with the boundary conditions imposing that the slope and the height vanish at the contact point:

$$
\begin{aligned}
& \phi_{1}^{(i)} \rightarrow 0, \quad \lambda_{1}^{(i)}=\mathcal{O}\left(s^{* 2}\right) \text { as } s^{*} \rightarrow 0^{+}, \\
& \phi_{1}^{(i)}=z_{1}^{(i)}=0, \quad \text { for } s^{*}=1 .
\end{aligned}
$$

Note that, unlike (3.27) or (3.47), the dependent variables $\left(z_{1}^{(i)}, \phi_{1}^{(i)}, \kappa_{1}^{(i)}, Q_{1}^{(i)}\right)$ are governed by a linear system $(3.61 a-d)$ that does not involve any term in the expansion of $T$ or $\lambda$. Physically, neither the shape of the vesicle nor its out-of-plane stress resultant are coupled, to leading order, with the stretch and in-plane strain.

Integrating (3.61 $a-d)$ using $(3.62 a-b)$ yields straightforwardly

$$
\begin{aligned}
z_{1}^{(i)} & =-\frac{1}{4} p_{0}^{(i)} H\left(s^{*}-1\right)\left[1+\left(1+s^{* 2}\right) \ln s^{*}-s^{* 2}\right], \\
\phi_{1}^{(i)} & =-\frac{1}{4} p_{0}^{(i)} H\left(s^{*}-1\right)\left[\frac{1}{s^{*}}+s^{*}\left(2 \ln s^{*}-1\right)\right], \\
Q_{1}^{(i)} & =p_{0}^{(i)} H\left(s^{*}-1\right) / s^{*}, \\
\lambda_{1}^{(i)} & =s^{* 2} / 8 .
\end{aligned}
$$


The growth of the leading-order variables $\phi_{1}^{(i)}, \kappa_{1}^{(i)}$ and, especially $\lambda_{1}^{(i)}$, as $s^{*} \rightarrow \infty$ indicates that the matching to the outer solution must be achieved through a second inner region, $\mathscr{R}_{(i i)}$, dominated by stretching. The solution in $\mathscr{R}_{(i i)}$ below will provide the two additional matching conditions which are required to determine $p_{0}^{(i)}$ and $T_{1}^{(i)}$.

3.5.2 Stretching region $\mathscr{R}_{(i i)}$, with length scale $\mathcal{O}\left(C^{-1 / 4}\right)$

We introduce a second inner region $\left(\mathscr{R}_{(i i)}\right)$ defined by the variable $\check{S}$ where $\tilde{S}=C^{-1 / 4} \check{S}$. This is done by re-scaling the leading-order dependent variables of case III (3.45) as

$$
\begin{aligned}
& \hat{z}_{1}=\frac{1}{2} \check{S}^{2}+z_{1}^{(i i)} / \ln \left(1 / \check{S}_{c}\right)+\cdots, \\
& \hat{\phi}_{1}=\check{s}+\phi_{1}^{(i i)} / \ln \left(1 / \check{S}_{c}\right)+\cdots, \\
& \hat{\kappa}_{1}=1+\kappa_{1}^{(i i)} / \ln \left(1 / \check{S}_{c}\right)+\cdots, \\
& \hat{Q}_{1}=Q_{1}^{(i i)} / \ln \left(1 / \check{S}_{c}\right)+\cdots, \\
& \hat{T}_{1}=T_{1}^{(i i)} / \ln \left(1 / \check{S}_{c}\right)+\cdots, \\
& \hat{\lambda}_{1}=\lambda_{1}^{(i i)} / \ln \left(1 / \check{S}_{c}\right)+\cdots,
\end{aligned}
$$

and taking the limit $\check{s}_{c} \rightarrow 0$ in (3.47)-(3.48), which then becomes

$$
\begin{aligned}
& z_{1 \check{s}}^{(i i)}=\phi_{1}^{(i i)}, \\
& \phi_{1 \check{s}}^{(i i)}=\kappa_{1}^{(i i)}, \\
& \kappa_{1 \check{s}}^{(i i)}=-\frac{\kappa_{1}^{(i i)}}{\check{s}}+\frac{\phi_{1}^{(i i)}}{\check{s}^{2}}-Q_{1}^{(i i)}, \\
& Q_{1 \check{s}}^{(i i)}=-\frac{Q_{1}^{(i i)}}{\check{s}}-2 T_{1}^{(i i)}, \\
& T_{1 \check{s}}^{(i i)}=-\phi_{1}^{(i i)}, \\
& \lambda_{1 \check{s}}^{(i i)}=-\phi_{1}^{(i i)}-2 \frac{\lambda_{1}^{I I}}{\check{s}},
\end{aligned}
$$

with the boundary conditions

$$
\begin{aligned}
& \phi_{1}^{(i i)} \sim \check{s} \log \check{s}, \quad z_{1}^{(i i)} \sim \frac{1}{2} \check{S}^{2} \log \check{s}, \quad \text { and } \quad \lambda_{1}^{(i i)} \rightarrow 0 \quad \text { as } \quad \check{s} \rightarrow 0^{+}, \\
& \phi_{1}^{(i i)} \rightarrow 0, \quad \text { and } \quad Q_{1}^{(i i)} \rightarrow 0 \quad \text { as } \quad \check{s} \rightarrow \infty
\end{aligned}
$$

derived from the matching conditions with the inner region $\mathscr{R}_{(i)}(3.63)$ as $\breve{s} \rightarrow 0^{+}$and with the outer region $\mathscr{D}_{\text {out }}^{+}$as $\breve{s} \rightarrow \infty$. Note that the first condition in (3.66a) removes two degrees of freedom and that the matching conditions with $\mathscr{R}_{(i)}$ for $\kappa_{1}^{(i i)}$ and $Q_{1}^{(i i)}, \kappa_{1}^{(i i)} \sim \log \check{S}$ 
(a)

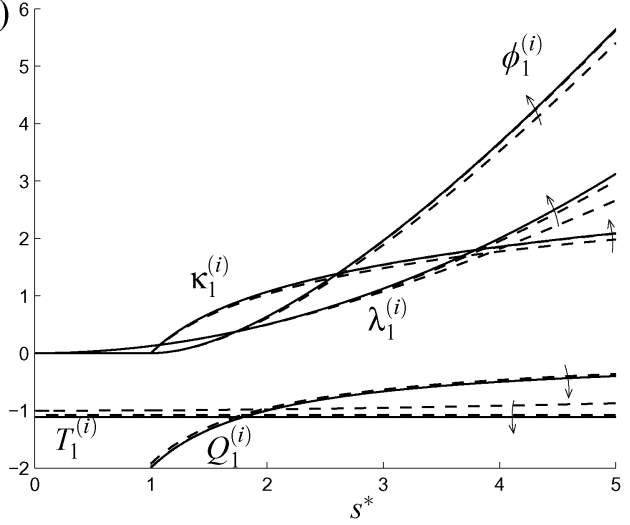

(b) 0.4

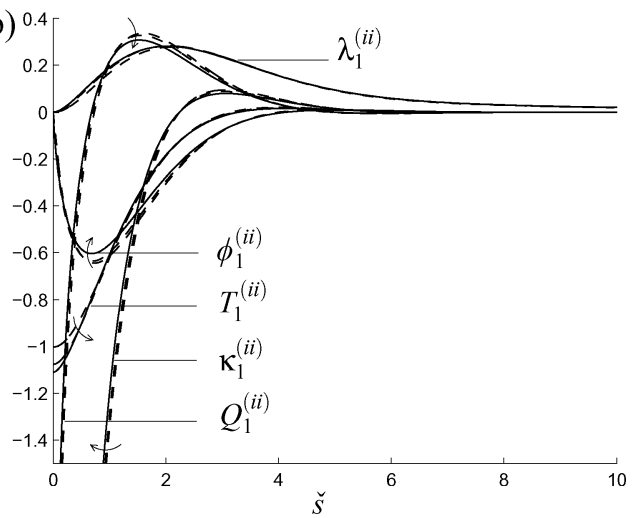

FIgURE 12. Case IV. Re-scaled tensions and shape of the vesicle for $\tilde{s}_{c} \ll C^{-1 / 4}$. Analytical solution (3.63)-(3.67) (solid lines) for case IV and numerical solution of (3.50)-(3.51) (dashed lines) for small values of $\check{s}_{c}$ in case III $\left(\check{s}_{c}=\left[10^{-1} ; 10^{-2}\right]\right)$. Arrows show decreasing values of $\check{s}_{c}$. (a) Region $\mathscr{R}_{(i)}$, where bending dominates; (b) region $\mathscr{R}_{(i i)}$, where stretching dominates.

and $Q_{1}^{(i i)} \sim-2 / \check{S}$ as $\check{S} \rightarrow 0$, are automatically satisfied. The solution of (3.65)-(3.66) is

$$
\begin{aligned}
& z_{1}^{(i i)}(\check{s})=-\frac{\pi \sqrt{2}}{4}+\frac{\pi \sqrt{2}}{4}\left[H_{0}^{(1)}\left(\frac{1+i_{\curlyvee}}{2^{1 / 4}}\right)+c . c .\right], \\
& \phi_{1}^{(i i)}(\check{s})=-a_{1} J_{1}\left(\frac{1+i_{\check{r}}}{2^{1 / 4}}\right)+\bar{a}_{1} Y_{1}\left(\frac{1+i_{\check{r}}}{2^{1 / 4}}\right)+c . c ., \\
& \kappa_{1}^{(i i)}(\check{s})=-\frac{\phi^{(i i)}(\check{s})}{\check{s}}-\frac{\pi}{2}\left[i H_{0}^{(1)}\left(\frac{1+i_{\check{r}}}{2^{1 / 4}}\right)+c . c .\right], \\
& Q_{1}^{(i i)}(\check{s})=\sqrt{2}\left[-a_{1} i J_{1}\left(\frac{1+i_{\check{L}}}{2^{1 / 4}} \check{s}\right)+i \bar{a}_{1} Y_{1}\left(\frac{1+i_{\check{L}}}{2^{1 / 4}}\right)+\text { c.c. }\right] \text {, } \\
& T_{1}^{(i i)}(\check{s})=-\frac{\pi \sqrt{2}}{4}\left[H_{0}^{(1)}\left(\frac{1+i_{\check{r}}}{2^{1 / 4}}\right)+\text { c.c. }\right] \text {, } \\
& \lambda_{1}^{(i i)}(\check{s})=\frac{\pi \sqrt{2}}{4}\left[J_{2}\left(\frac{1+i}{2^{1 / 4}} \check{s}\right)-I_{2}\left(\frac{1+i^{\prime}}{2^{1 / 4}} \check{s}^{\prime}\right)\right] \\
& +\frac{\pi}{\breve{S}^{2}}\left[G_{24}^{21}\left(\frac{i \check{S}^{2} \sqrt{2}}{4} \mid \begin{array}{cccc}
1 & \frac{1}{2} & & \\
2 & 1 & \frac{1}{2} & 0
\end{array}\right)+\text { c.c. }\right] \text {, }
\end{aligned}
$$

where $I_{n}, J_{n}$ and $Y_{n}$ are Bessel functions, $H_{0}^{(1)}$ is a Hankel function, $G_{p q}^{m n}$ are Meijer $G$-functions and $a_{1} \equiv(1+i) \pi 2^{-7 / 4} ;$ c.c. denotes complex conjugate.

The solution (3.67) is plotted in Figure 12(b) and compared with the numerical solutions of (3.50)-(3.51) for case III (re-scaled accordingly). There is good agreement as $\check{s}_{c} \rightarrow 0$.

Following $(3.67 b, e)$, the matching conditions between regions $\mathscr{R}_{(i)}$ and $\mathscr{R}_{(i i)}$ yield the following conditions on $\phi_{1}^{(i)}$ and $T_{1}^{(i)}$ :

$$
\phi_{1}^{(i)} \sim s^{*} \ln s^{*} \quad \text { and } \quad T_{1}^{(i)} \sim-\pi \sqrt{2} / 4 \text { as } s^{*} \rightarrow \infty,
$$

which imply $T_{1}^{(i)}\left(s^{*}\right)=-\pi \sqrt{2} / 4$ and $p_{0}^{(i)}=-2$, i.e. $\check{p}_{0}=-2 / \check{S}_{c} \ln \left(1 / \check{S}_{c}\right)$ (see crosses in 
Figure 8). To leading order as $\tilde{s}_{c} \ll C^{-1 / 4} \ll 1$, following (2.12), we thus have

$$
p=\frac{-2 C^{1 / 2}}{\check{S}_{c} \ln \left(1 / \check{S}_{c}\right)} \delta\left(\check{s}-\check{s}_{c}\right) \quad \text { and } \quad F=\frac{4 \pi}{\ln \left(1 / \check{S}_{c}\right)} .
$$

The solutions (3.63) agree very well with those obtained by solving (3.47)-(3.48) numerically for small values of $\check{s}_{c}$ (Figures $8(\mathrm{a})$ and (b) and Figure 12(a)).

Accounting for the correction brought by (3.67), the corrective terms for the shape of the vesicle in the outer region $\mathscr{D}_{\text {out }}^{+}$are

$$
\bar{z}_{1}^{+}=0 \quad \text { and } \quad \bar{z}_{2}^{+}=\frac{1}{\ln \left(1 / \check{s}_{c}\right)} \int_{0}^{\infty} \phi_{1}^{(i i)} \mathrm{d} t .
$$

Using (3.65e) and $(3.67 d)$, we have $\int_{0}^{\infty} \phi_{1}^{(i i)} \mathrm{d} \hat{t}=\lim _{\check{s} \rightarrow 0} T_{1}^{(i i)}(\check{s})=-\pi \sqrt{2} / 4$. The terms in the expansion of $h(3.1)$ are then

$$
h_{0}=2, \quad h_{1}=0 \quad \text { and } \quad h_{2}=2 \bar{z}_{2}^{+}=\frac{-\pi \sqrt{2}}{2 \ln \left(1 / \check{s}_{c}\right)},
$$

so that writing the expression for the force derived in (3.69) in terms of the plates' displacement $2-h=-C^{-1 / 2} h_{2}$ yields

$$
F=4 \sqrt{2} C^{1 / 2}(2-h)
$$

Thus while $F$ is singular in $\tilde{s}_{c}$ (Figure $8(\mathrm{~b})$ ), it is linear in $2-h$. Inverting (3.71), the radius of the contact disk is then given in term of the plates' displacement by

$$
\tilde{s}_{c}=C^{-1 / 4 \check{S}_{c}}=C^{-1 / 4} \exp \left(-\frac{\pi}{\sqrt{2} C^{1 / 2}(2-h)}\right) .
$$

\section{Discussion}

In continuation to the work of Preston et al. (2008), we considered the static deformation of an axisymmetric vesicle in compression between parallel plates. The vesicle is modelled using thin-shell theory and constitutive laws proposed by Evans \& Skalak (1980) and Pamplona \& Calladine (1993). In this framework, the mechanical properties of the vesicle are described by a single parameter $C$ that measures the ratio of the vesicle's resistance to shear to its resistance to bending.

In general, the problem to be solved is a sixth-order boundary-value problem (2.11) with an internal free boundary $\tilde{s}_{c}$ (the position of the contact point, as defined in Figure 1) and two independent parameters, $h$ (the inter-plate distance) and $C$. We investigated the asymptotic limit $C \gg 1$ and, for mathematical convenience, we treated $\tilde{s}_{c}$ as a control parameter and $h$ as an unknown parameter. In this parameter space, two families of solutions exist, corresponding to an unbuckled vesicle (which is flattened at its poles) and a buckled vesicle (with reverse curvature at the poles, Figure 3). The existence and stability 
of each solution, as well as their mechanical behaviour, depend on both $\tilde{s}_{c}$ and $C$. Much physical insight can be gained by exploring four different zones in the $\left(C, \tilde{s}_{c}\right)$-parameter space, labelled as cases I-IV in Figure 7.

Along any meridional cross-section, the vesicle exhibits different spatial regions, each of which is characterised by a distinct balance of forces. As anticipated from prior studies of buckled shells (Landau \& Lifshitz, 1986; Pogoroelov, 1986), in an (inner) region of width $\mathcal{O}\left(C^{-1 / 4}\right)$ around the contact point, contact stresses are balanced by both resistance to bending and resistance to shear (i.e. stretching) to leading order. Everywhere else (i.e. in the outer regions), the statics of the vesicle are dominated by high resistance to shear, resulting in solutions that locally resemble sectors of spheres (Figure 3). However, for very small deformations (case IV), there exists an $\mathcal{O}\left(\tilde{S}_{c}\right)$ region around the pole dominated by resistance to bending where stretching is negligible.

In the distinguished limit $C^{-1 / 4} \ll \tilde{S}_{c} \sim 1$ (case I), (2.11) reduces to a fifth-order local inner problem (3.22) for the stretching-bending boundary layer. The latter is only marginally simpler than the original sixth-order problem and we did not seek to solve it explicitly here. In this regime, constitutive terms coupling bending and stretching effects (Pamplona et al., 2005) are expected to have a quantitative leading-order effect (Appen$\operatorname{dix} \mathrm{A})$.

A further simplification assuming that $C^{-1 / 4} \ll \tilde{s}_{c} \ll 1$ (case II) yields a remarkably simple autonomous fourth-order problem (3.29) (identified using a different constitutive law, and under different loading conditions, by Parker \& Wan (1984)) that applies across a wide range of the parameter space. Equation (3.29) can be solved numerically, enabling us to determine the shape and stresses of the post-buckled vesicle. The force-displacement relation for the buckled solution can be captured only by going to higher order in the expansion, a feature seen also in the analysis of e.g. Kriegsmann \& Lange (1980). This is achieved straightforwardly for $C^{-1 / 4} \ll \tilde{S}_{c} \ll C^{-1 / 12}$ (case IIb, which represents a large subset of case II), where the quadratic force-displacement relation (3.44) is derived as a solvability condition. We find that, for $C^{-1 / 2} \ll 2-h \ll C^{-1 / 6}$, the magnitude of the delta-function representing the transmural pressure $p \approx-3.97 C^{1 / 4} \delta(\tilde{s}-\sqrt{2-h})$ does not depend on $h$, so that the contact force is proportional to the circumference of the contact ring: $F \approx 24.9 C^{1 / 4} B a^{-1} \sqrt{2-h / a}$ to leading order in dimensional variables (Figure 6).

The second distinguished limit $\tilde{s}_{c} \sim C^{-1 / 4}$ (case III) leads to a non-autonomous fourth-order problem (3.50) that can be solved numerically, capturing both the onset of the buckling instability (Figures 7(e) and 10) and the leading-order force-displacement relation (Figure 13). In dimensional variables, we find $F=B a^{-1} f$, where $f$ is an $\mathcal{O}(1)$ function of $C^{1 / 2}(2-h / a)$ which is plotted in Figure 13(a). These results are generic and provide a universal description of the leading-order behaviour of a spherical vesicle under compression with this set of constitutive relations (2.3)-(2.7). The scalings for the asymptotic pre- and post-buckled force-displacement relations (case II above and IV below) when $f$ is quadratic and linear respectively, agree with those derived by Komura et al. (2005) and Fery \& Weinkamer (2007) using scaling arguments that balance bending and stretching energies.

For $C^{-1 / 2} \ll \tilde{s}_{c} \ll C^{-1 / 4}$ (case IV), (2.11) reduces to a non-autonomous fourthorder (3.61) and fifth-order problem (3.65), corresponding respectively to an $\mathcal{O}\left(\tilde{s}_{\mathcal{C}}\right)$ region 

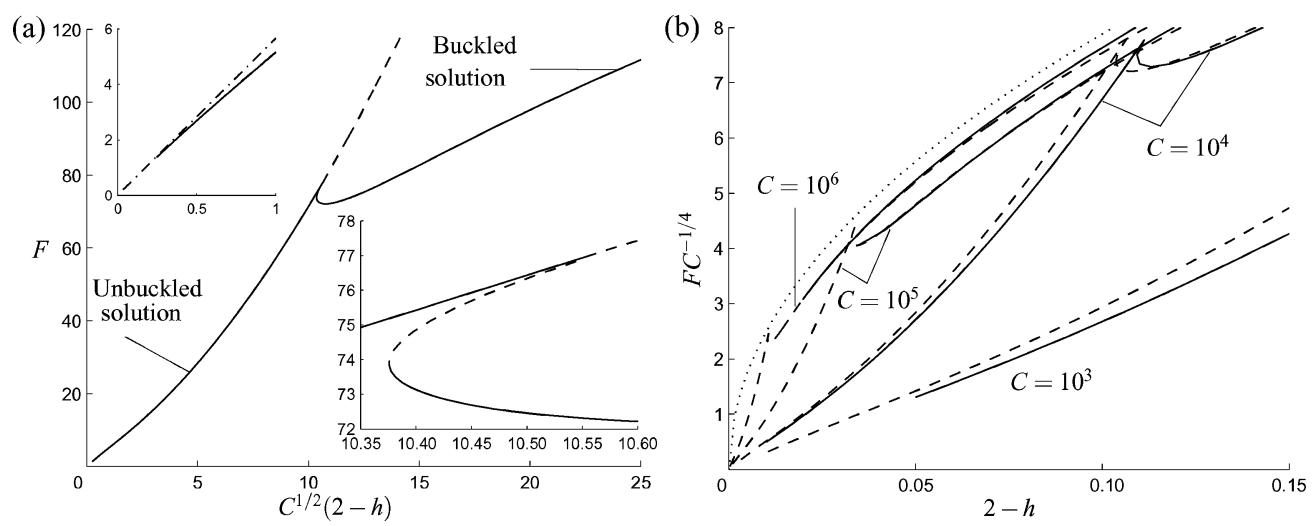

FIGURE 13. Non-dimensional force $F$ versus plate displacement $2-h$. (a) 'Universal' asymptotic relationship obtained in case III from the solution of (3.47) and (3.53). The lower inset is a magnified view of the sub-critical buckling instability. The upper inset shows the region $C^{1 / 2}(2-h) \ll 1$ and the asymptote (3.72) (dot-dashed) found for case IV. (b) Leading-order asymptotic solutions from case III (dashed lines) compared with numerical solutions of the full system (2.11)-(2.13) (solid) for $C=\left[10^{3} ; 10^{4} ; 10^{5} ; 10^{6}\right]$. The dotted line corresponds to asymptotic results for the post-buckled state in case II (3.44).

dominated by bending and a larger $\mathcal{O}\left(C^{1 / 4}\right)$ region dominated by stretching. An analytical solution can be found that satisfies the matching conditions with the $\mathcal{O}(1)$ outer region, thereby providing validation of the pre-buckled solution in the limit of small deformations (see the insets in Figures 8(b) and 13(a)). In this case, the leading-order force-displacement relation is linear and, in dimensional variables, we find $F=4 \sqrt{2} C^{1 / 2} B a^{-1}(2-h / a)$. This linear relationship masks a logarithmic dependence of $F$ on $\tilde{s}_{c}$ (3.69). Although bending and stretching here operate on different length scales, it is notable that the scaling for $F$ can still be obtained by balancing these two energies (Komura et al., 2005). Case IV shows that stress-focusing may not always occur in the limit $C \gg 1$.

As the dimensionless distance $h$ between the plates is decreased from its initial value of 2 (the diameter of the vesicle in its initial spherical configuration), the contact between the vesicle and the lower plate (say) changes from a point (for $h=2$ ) to a disk (for $h_{\mathrm{TC}} \leqslant h \leqslant 2$; see also (3.73)) and then to a ring of radius $r_{c}$ (for $h<h_{\mathrm{TC}}$ ) as the vesicle buckles. The critical displacement $h_{\mathrm{TC}}$ for which the buckling occurs (i.e. the transcritical bifurcation point) is found to be $h_{\mathrm{TC}} \approx 2-10.56 C^{-1 / 2}$. The buckling instability is found to be sub-critical (in our analysis, this feature arises from the non-linear relation between $\tilde{S}_{c}$ and $h$, at $\mathcal{O}\left(C^{-1 / 2}\right)$ ), with a saddle-node bifurcation point $h_{S N} \approx 2-10.38 C^{-1 / 2}$ (see Figure 13(a)). This is again consistent with the values obtained numerically by Preston et al. (2008).

Away from an inner region of $\mathcal{O}\left(C^{-1 / 4}\right)$ around the contact point in the post-buckled state, we find that, to leading order, the shape of the compressed vesicle is that of the initial sphere except that the caps at the poles described by $\tilde{s}<\tilde{s}_{c}$ now point inwards as in Figure 1. The stresses to which the vesicle is subject are left unchanged to leading order. The next order correction to the shape of the vesicle is $\mathcal{O}\left(C^{-3 / 2}\right)$ in $\mathscr{D}_{\text {out }}^{-}$and $\mathcal{O}\left(C^{-3 / 4}\right)$ in 
$\mathscr{D}_{\text {out }}^{+}$. For instance, at the equator, the radius of the vesicle varies like $1+3.97 \sqrt{2-h} C^{-3 / 4}$ for $C^{-1 / 2} \ll 2-h \ll C^{-1 / 6}$ (case IIb).

Most of the changes in the dependent variables occur within the inner region. To leading order, and for $C^{-1 / 2} \ll 2-h \ll 1$ (case II), the shape and tensions of the vesicle are continuous across the whole domain and can be described by a self-similar structure with the location of the contact point $\tilde{s}_{c}$ (or equivalently $\sqrt{2-h}$, in this case) as a scaling variable. The stresses in the vesicle (and its shape) are then determined only by the distance between the plates. Moreover, the out-of-plane stress resultant $Q$ is found to be $\mathcal{O}\left(C^{1 / 2}\right)$ and continuous across the contact point (to leading order). This result is not very intuitive and implies that the contact forces acting on the vesicle, through the $\mathcal{O}\left(C^{1 / 4}\right)$ pressure term, do not contribute to leading order.

Interestingly, our results confirm analytically that $Q \rightarrow 0$ at the left-hand side of the discontinuity $\tilde{s}=\tilde{s}_{c}$ (to first and second order for $C \gg 1$ ). This is consistent with solutions obtained by solving the full problem numerically, which exhibited $Q \rightarrow 0$ as $\tilde{s} \rightarrow \tilde{s}_{c}^{-}$to within the accuracy of the numerical method (see Figure 5(e) and Preston et al. (2008) for details). This feature is not imposed explicitly by the boundary conditions and was left unexplained by Preston et al. (2008). This result can be recovered from physical arguments by noting that the net vertical force acting on the inside region $0 \leqslant \tilde{s}<\tilde{s}_{c}$, which is proportional to $\lim _{\tilde{s} \rightarrow \tilde{s}_{c}^{-}} Q$ (since the vanishing slope in this region implies that the in-plane tension does not contribute to the vertical force balance) and excludes the point of contact with the plate, must be zero.

Arguably, our asymptotic analysis, involving powers of $C^{-1 / 4}$, requires large values of $C$ for convergence. However, the good quantitative agreement, for finite values of $C$ within the range $10^{3}-10^{6}$, between the asymptotics and the full numerical results (Figures 5 and 13) suggests that the physical insight gained from our analysis should be applicable to a wide range of materials. This includes, for example, ping-pong or tennis balls (respectively $C \approx 10^{4}$ and $C \approx 10^{3}$ using the mechanical description of capsules by Pozrikidis (2003) and the parameters measured by Pauchard \& Rica (1998)) or lipid bilayer membranes (typical values for red blood cells are $C \approx 100$ (Noguchi \& Gompper, 2005)). Of course, under sufficiently large loading, we can expect the axisymmetric dimpled states computed here to undergo a secondary bifurcation to non-axisymmetric configurations (Pauchard \& Rica, 1998; Gupta et al., 2008; Vaziri \& Mahadevan, 2008) which fall outside the present modelling framework.

By using shell theory to describe the vesicle, we assume that its thickness is less than any length scale identified in Figure 7. Writing the Föppl-von Kármán number (in the framework of linear elasticity) as $C=12\left(1-v^{2}\right) a^{2} / h_{s}^{2}$ for a membrane of thickness $h_{s}$ and Poisson ratio $v$ (Landau \& Lifshitz, 1986), this assumption is straightforwardly satisfied for cases I, II and III. In case IV, it requires $h_{s} \propto C^{-1 / 2} \ll \tilde{S}_{c}$ and thus, using (3.73), sets a lower bound $2-h \gg \pi C^{-1 / 2} / \sqrt{2} \ln C^{1 / 4}$ on the plate displacement that we can consider in this paper (as indicated in Figure 7). This limiting case is considered by Gregory et al. (1998). The behaviour of the vesicle under different loading conditions, the effects of friction and membrane permeability, as well as the transition to asymmetric buckling, remain to be addressed. However, the approximations identified here provide a useful foundation for such investigations. 


\section{Acknowledgements}

The contributions of Dr. Simon Preston to the early stages of this study are gratefully acknowledged.

\section{Appendix A Effects of the ' $M \kappa$ ' term}

Here we investigate the effects of an additional constitutive term coupling bending and stretching effects, proposed by Pamplona et al. (2005), and show that for $C \gg 1$ it is significant only for strongly deformed vesicles.

In place of (2.5), we assume that the principal tensions and stretches are related by

$$
N_{\phi}-N_{\theta}=H\left(\lambda^{-1}-\lambda\right)+M_{\phi}\left(\kappa_{\theta}-\kappa_{\phi}\right)
$$

We also include the contribution of the spontaneous curvature $\kappa_{0}$ into the second constitutive equation (2.7):

$$
M_{\phi}=M_{\theta}=B\left(\kappa_{\phi}+\kappa_{\theta}-2 \kappa_{0}\right) \equiv M
$$

We then decompose the principal in-plane stress resultants in terms of the isotropic stress resultant $T$ so that (A 1) becomes

$$
N_{\phi}=T+H / \lambda-M \kappa_{\phi} \quad \text { and } \quad N_{\theta}=T+H \lambda-M \kappa_{\theta}
$$

After non-dimensionalisation (2.8), with $\kappa \equiv \kappa_{\phi}$ and $\kappa_{\theta}=\sin \phi / r$, (A 2) and (A 3) become

$$
N_{\phi}=T+\frac{1}{\lambda}-\frac{M}{C} \kappa, \quad N_{\theta}=T+\lambda-\frac{M}{C} \frac{\sin \phi}{r}, \quad M=\kappa+\frac{\sin \phi}{r}-2 \kappa_{0} .
$$

The $1 / C$ pre-factors indicate the weak effects of the new terms in the large $C$-limit, as we now explain in more detail.

\section{A.1 Governing equations}

With the new terms, the governing system of dimensionless ODEs (2.11) are replaced by

$$
\begin{aligned}
r_{\tilde{s}}= & \cos \phi / \lambda, \\
z_{\tilde{s}}= & \sin \phi / \lambda, \\
\phi_{\tilde{s}}= & \kappa / \lambda, \\
\kappa_{\tilde{s}}= & {\left[-Q+\sin \phi \cos \phi / r^{2}-\kappa \cos \phi / r\right] / \lambda, } \\
Q_{\tilde{s}}= & {[p-C(T+1 / \lambda) \kappa-C(T+\lambda) \sin \phi / r-Q \cos \phi / r] / \lambda } \\
& +\left(\sin \phi / r+\kappa-2 \kappa_{0}\right)\left(\sin ^{2} \phi / r^{2}+\kappa^{2}\right) / \lambda, \\
C T_{\tilde{s}}= & {\left[C \lambda_{\tilde{s}} / \lambda+C(\lambda-1 / \lambda) \cos \phi / r+\kappa Q\right] / \lambda } \\
& +\left[\kappa\left(\sin \phi / r+\kappa-2 \kappa_{0}\right)\right]_{\tilde{s}}+\cos \phi\left(\sin \phi / r+\kappa-2 \kappa_{0}\right)(\kappa-\sin \phi / r) / r \lambda, \\
\lambda= & r / \sin \tilde{s},
\end{aligned}
$$


while the equation for $N_{\phi}$ becomes

$$
N_{\phi}=T+\frac{1}{\lambda}-\frac{\kappa}{C}\left(\frac{\sin \phi}{r}+\kappa-2 \kappa_{0}\right) .
$$

Note that with the exception of the terms on the second lines of (A $5 e$ ) and (A $5 f$ ), which arise from the $M \kappa_{\phi}$ and $M \kappa_{\theta}$ terms in (A 3), equations (A 5) are identical to (2.11). The boundary conditions (2.13) are unchanged.

\section{A.2 Asymptotics}

\section{A.2.1 Outer region}

After substitution of (3.2) in (A 5), the new terms appear at $\mathcal{O}\left(C^{-1}\right)$ in (A $5 e$ ) and (A $5 f$ ), and thus do not affect the first two orders of the outer solution, namely (3.3) and (3.9).

\section{A.2.2 Inner region, case I (i.e. $C^{-1 / 4} \ll \tilde{s}_{c} \sim 1$ )}

We define $\hat{N}_{1}$ as in Section 3.2, i.e. $\hat{N}_{1} \equiv \hat{T}_{1}-\hat{\lambda}_{1}+\hat{\lambda}_{0}^{2}$. Note that (3.20) becomes

$$
N_{\phi}=\left(\hat{T}_{0}-\hat{\lambda}_{0}\right) C^{-1 / 4}+\left[\hat{N}_{1}-\hat{\kappa}_{1}^{2}\right] C^{-1 / 2}+\cdots .
$$

Identifying terms of $\mathcal{O}(C)$ in (A 5) and using the expansion (3.19) yields $\hat{T}_{0}=\hat{\lambda}_{0}$, as in Section 3.2. Proceeding to $\mathcal{O}\left(C^{3 / 4}\right)$ leads to amended versions of (3.21bii) and (3.21cii)

$$
\begin{aligned}
& \hat{Q}_{1 \hat{s}}=-\hat{N}_{1} \hat{\kappa}_{1}-2 \hat{\lambda}_{0} \sin \hat{\phi}_{1} / \sin \tilde{s}_{c}+\hat{\kappa}_{1}^{3}, \\
& \hat{N}_{1 \hat{s}}=2 \hat{\lambda}_{0} \cos \hat{\phi}_{1} / \sin \tilde{s}_{c}+\hat{\kappa}_{1} \hat{Q}_{1}+\left(\hat{\kappa}_{1}^{2}\right)_{\hat{s}},
\end{aligned}
$$

where the new terms are in each case the final ones of the right-hand side. In turn, these lead to an amended version of (3.22),

$$
\begin{aligned}
\hat{\phi}_{1 \hat{s} \hat{s} \hat{s}} & =\hat{N}_{1} \hat{\phi}_{1 \hat{s}}+2 \hat{\lambda}_{0} \sin \hat{\phi}_{1} / \sin \tilde{s}_{c}-\hat{\phi}_{1 \hat{s}}^{3}, \\
\hat{N}_{1 \hat{s}} & =2 \hat{\lambda}_{0} \cos \hat{\phi}_{1} / \sin \tilde{s}_{c}+\hat{\phi}_{1 \hat{s}} \hat{\phi}_{1 \hat{s} \hat{s}}, \\
\hat{\lambda}_{0 \hat{s}} & =\left(\cos \hat{\phi}_{1}-\cos \tilde{s}_{c}\right) / \sin \tilde{s}_{c},
\end{aligned}
$$

with boundary conditions unchanged from (3.23). Here the new terms are the final ones of $(\mathrm{A} 7 a)$ and $(\mathrm{A} 7 b)$.

The new terms in (A 5) have thus introduced some additional non-linear terms in the leading-order system (3.22), which would obviously affect the solution. The effects are presumably limited to $\hat{S}=\mathcal{O}(1)$ since linearising (A 7) about the far-field limits leads to solutions of the same form as (3.25) for $\hat{s} \rightarrow \pm \infty$.

We do not solve (A 7) but show that the additional terms become higher order as $\tilde{s}_{c} \ll 1$. 
A.2.3 Inner region, case II (i.e. $C^{-1 / 4} \ll \tilde{S}_{c} \ll 1$ )

After substitution of the expansion (3.26) in (A 5), the non-linear $M \kappa$ terms are $\mathcal{O}\left(C^{3 / 4} \tilde{s}_{c}^{3}\right)$ and therefore vanish under the restriction $\tilde{s}_{c} \ll C^{-1 / 12}$ (see Section 3.3.1). They appear in the solvability condition (3.43) (with other non-linearities) only when $\tilde{s}_{c} \sim C^{-1 / 12}$ (a case we do not consider explicitly).

\section{A.2.4 Inner region, cases $I I I$ and $I V$}

For cases III and IV, the contributions of the $M \kappa$ terms are $\mathcal{O}\left(C^{1 / 2}\right)$ smaller than the leading-order terms, and we recover the leading-order equations (3.47), (3.61) and (3.65).

\section{References}

Blyth, M. G. \& Pozrikidis, C. (2004) Solution space of axisymmetric capsules enclosed by elastic membranes. Eur. J. Mech. - A/Solids 23, 877-892.

Canham, P. B. (1970) The minimum energy of bending as a possible explanation of the biconcave shape of the human red blood cell. J. Theor. Biol. 26, 61-81.

Evans, E. A. \& SkalaK, R. (1980) Mechanics and Thermodynamics of Biomembranes, CRC Press. Boca Raton, Florida, 97-112.

Evkin, A. Y. \& Kalamkarov, A. L. (2001) Analysis of large deflection equilibrium states of composite shells of revolution. Part 1. General model and singular perturbation analysis. Int. J. Solids Struct. 38, 8961-8974.

Fery, A. \& Weinkamer, R. (2007) Mechanical properties of micro- and nanocapsules: Single-capsule measurements. Polymer 48, 7221-7235.

Graff, M., Scheidl, R., Troger, H. \& Weinmuller, E. (1985) An investigation of the complete postbuckling behavior of axisymmetrical spherical-shells. ZAMP 36, 803-821.

Gregory, R. D., Milac, T. I. \& Wan, F. Y. M. (1998) A thick hollow sphere compressed by equal and opposite concentrated axial loads: An asymptotic solution. SIAM J. Appl. Math. 59, 1080-1097.

Gupta, N. K., Sheriff, N. M. \& Velmurugan, R. (2008) Experimental and theoretical studies on buckling of thin spherical shells under axial loads. Int. J. Mech. Sci. 50, $422-432$.

Helfer, E., Harlepp, S., Bourdieu, L., Robert, J., MacKintosh, F. C. \& Chatenay, D. (2001) Buckling of actin-coated membranes under application of a local force. Phys. Rev. Lett. 87, 088103.

Huang, Y. Q., Doerschuk, C. M. \& Kamm, R. D. (2001) Computational modeling of $\mathrm{RBC}$ and neutrophil transit through the pulmonary capillaries. J. Appl. Physiol. 90, 545-564.

Komura, S., Tamura, K. \& Kato, T. (2005) Buckling of spherical shells adhering onto a rigid substrate. Eur. Phys. J. E - Soft Matter 18, 343-358.

Kriegsmann, G. A. \& Lange, C. G. (1980) On large axisymmetrical deflection states of spherical shells. J. Elast. 10, 179-192.

Kumar, M. N. R. (2000) Nano and microparticles as controlled drug delivery devices. J. Pharm. Sci. 3, 234-258. 
Landau, L. D. \& Lifshitz, E. M. (1986) Theory of Elasticity, 3rd ed., Pergamon, New York 7, 54-57.

Lidmar, J., Mirny, L. \& Nelson, D. R. (2003) Virus shapes and buckling transitions in spherical shells. Phys. Rev. E Stat. Nonlinear Soft Matter Phys. 68, 051910.

An asymptotic analysis of the buckling of a highly shear-resistant vesicle

Lobkovsky, A. E. \& Witten, T. A. (1997) Properties of ridges in elastic membranes. Phys. Rev. E 55, 1577-1589.

Monllor, P., Bonet, M. A. \& CASEs, F. (2007) Characterization of the behaviour of flavour microcapsules in cotton fabrics. Eur. Polym. J. 43, 2481-2490.

Murray, F. J. \& Wright, F. W. (1961) The buckling of thin spherical shells. J. Aerosp. Sci. 28, 223-236.

Noguchi, H. \& Gomprer, G. (2005) Shape transitions of fluid vesicles and red blood cells in capillary flows. Proc. Natl. Acad. Sci. USA 102, 14159-14164.

Pamplona, D. C. \& Calladine, C. R. (1993) The mechanics of axially symmetric liposomes. J. Biomech. Eng. 115, 149-159.

Pamplona, D. C., Greenwood, J. A. \& Calladine, C. R. (2005) The buckling of spherical liposomes. J. Biomech. Eng. 127, 1062-1069.

PARKER, D. F. \& WAN, F. Y. M. (1984) Finite polar dimpling of shallow caps under sub-buckling axisymmetric pressure distributions. SIAM J. Appl. Math. 44, 301326.

PARker, K. H. \& Winlove, C. P. (1999) The deformation of spherical vesicles with permeable, constant-area membranes: Application to the red blood cell. Biophys. J. 77, 3096-107.

PAuchard, L. \& Rica, S. (1998) Contact and compression of elastic spherical shells: The physics of a ping-pong ball. Phil. Mag. B 78(9), 225-233.

Pogoroelov, A. V. (1986) Bendings of Surfaces and Stability of Shells. Translations of Mathematical Monographs Vol. 72, American Mathematical Society, Providence, RI.

PozRikidis, C. (2003) Shell theory for capsules and cells. Ch. 2 (pages 35-101) of Modelling and Simulation of Capsules and Biological Cells, ed. Pozrikidis, C., CRC Press, Boca Raton, Florida.

Preston, S. P., Jensen, O. E. \& Richardson, G. (2008) Buckling of an axisymmetric vesicle under compression: The effects of resistance to shear. Q. J. Mech. Appl. Math. 61, 1-24.

Risso, F. \& CARIN, M. (1987) Compression of a capsule: Mechanical laws of membranes with negligible bending stiffness. Phys. Rev. E 69, 061601.

Scheidl, R. \& Troger, H. (1987) A comparison of the postbuckling behavior of plates and shells. Comput. Struct. 27, $157-163$.

Schrooyen, P. M., van Der Meer, R. \& Kruif, C. G. D. (2001) Microencapsulation: Its application in nutrition. Proc. Nutr. Soc. 60, 475-479.

Schwarz, U. (2007) Soft matters in cell adhesion: Rigidity sensing on soft elastic substrates. Soft Matter 3, 263-266.

Smith, A. E., Moxham, K. E. \& Middelberg, A. P. J. (1998) On uniquely determining cell-wall material properties with the compression experiment. Chem. Eng. Sci. 53, 3913-3922. 
VAZIRI, A. \& Mahadevan, L. (2008) Localized and extended deformations of elastic shells. Proc. Natl. Acad. Sci. 105, 7913-7918.

Wan, F. Y. M. (1980) The dimpling of spherical caps. Mech. Today 5, 495-508.

Wan, K., Chan, V. \& Dillard, D. A. (2003) Constitutive equation for elastic indentation of a thin-walled bio-mimetic microcapsule by an atomic force microscope tip. Colloids Surf. B: Biointerf. 27, 241-248.

Witten, T. A. (2007) Stress focusing in elastic sheets. Rev. Mod. Phys. 79, 643-33.

Zarda, P. R., Chien, S. \& Skalak, R. (1977) Elastic deformations of red blood cells. J. Biomech. 10, 211-21. 